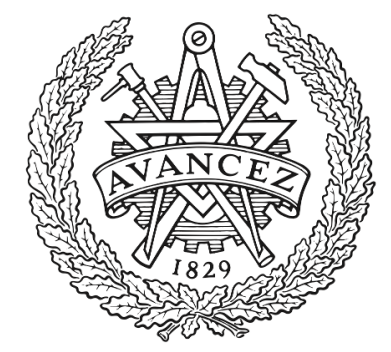

\title{
CHALMERS
}

UNIVERSITY OF TECHNOLOGY

\section{On a volume averaged measure of macroscopic reinforcement slip in two-scale modeling of reinforced concrete}

Downloaded from: https://research.chalmers.se, 2023-04-26 00:22 UTC

Citation for the original published paper (version of record):

Sciegaj, A., Larsson, F., Lundgren, K. et al (2020). On a volume averaged measure of macroscopic reinforcement slip in two-scale modeling of reinforced concrete. International Journal for Numerical Methods in Engineering, 121(8): 1822-1846. http://dx.doi.org/10.1002/nme.6288

N.B. When citing this work, cite the original published paper. 
This is the accepted version of the following article:

Sciegaj A, Larsson F, Lundgren K, Runesson K. On a volume averaged measure of macroscopic reinforcement slip in two-scale modeling of reinforced concrete. International Journal for Numerical Methods in Engineering. 2020; 121(8): 1822-1846

which has been published in final form at https://doi. org $/ 10.1002 /$ nme. 6288 .

This article may be used for non-commercial purposes in accordance with the Wiley Self-Archiving Policy https: //authorservices.wiley.com/author-resources/ Journal-Authors/licensing-open-access/ open-access/self-archiving.html

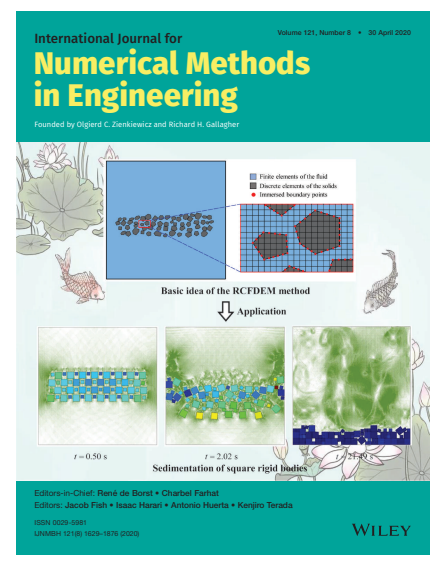




\title{
On a volume averaged measure of macroscopic reinforcement slip in two-scale modelling of reinforced concrete
}

\author{
Adam Sciegaj $^{\mathrm{a}, \mathrm{b}, *}$, Fredrik Larsson ${ }^{\mathrm{b}}$, Karin Lundgren $^{\mathrm{a}}$, Kenneth Runesson ${ }^{\mathrm{b}}$ \\ ${ }^{a}$ Department of Architecture and Civil Engineering, Chalmers University of Technology, Gothenburg, \\ Sweden \\ ${ }^{b}$ Department of Industrial and Materials Science, Chalmers University of Technology, Gothenburg, Sweden
}

\begin{abstract}
A two-scale model for reinforced concrete, in which the large-scale problem formulation is enriched by an effective reinforcement slip variable, is derived from the single-scale model describing the response of plain concrete, reinforcement steel, as well as the bond between them. The subscale problem on the Representative Volume Element (RVE) is correspondingly defined as finding the response of the RVE subjected to effective variables (strain, slip, and slip gradient) imposed from the large-scale. A novel volumetric definition of effective reinforcement slip and its gradient is devised, and the corresponding subscale problem is formulated. The newly-defined effective variables are imposed on the RVE in a weak sense via Lagrange multipliers. The response of the RVEs of different sizes was investigated by means of pull-through tests, and the novel boundary condition type was used in $\mathrm{FE}^{2}$ analyses of a deep beam. Locally, prescribing the macroscopic reinforcement slip and its gradient in the proposed manner resulted in reduced RVE-size dependency of effective work conjugates, which allows for more objective description of reinforcement slip in two-scale modelling of reinforced concrete. Globally, this formulation produced more consistent amplitudes of effective slip fluctuations, as well as more consistent maximum crack width predictions.
\end{abstract}

Keywords: reinforced concrete, bond-slip, multiscale, computational homogenisation, cracking, Lagrange multipliers

\section{Introduction}

The brittle nature of concrete, resulting in cracking even at moderate load levels, is a factor that significantly influences the durability of the structure. Cracks on their own need not necessarily result in structural failure, but rather pave the way for ingress of harmful substances causing reinforcement corrosion [1, 2, 3]. Hence, it is important to be able to model crack growth process in detail and the bond action between the concrete and reinforcement need to be included $[4,5]$.

\footnotetext{
* Corresponding author

Email address: adam.sciegaj@chalmers.se (Adam Sciegaj)
} 
The bond action in reinforced concrete can be ascribed to different mechanisms, friction being the most important. Several modelling approaches exist, with so-called bond-slip models being particularly popular recently. These models describe the relation between the frictional stresses along the reinforcement (called bond stresses) and the difference in deformation between steel and concrete (called the reinforcement slip). The bond-slip relation depends on many factors, such as e.g. the type and shape of the reinforcement, confinement, concrete properties etc., but can in many cases be successfully used in modelling [6]. Bond-slip models have been widely used for modelling the response of reinforced concrete structures. The effect of bond can be modelled in different ways, one option being suitable enrichment of finite elements [7, 8, 9] based on extended finite element method or embedded discontinuity formulation. Another popular option, available in most commercial codes is to directly resolve the interface around the reinforcement with interface/cohesive zone elements. Since interface elements are usually included in the commercial codes, this option is often preferred in practice, as it does not require any additional implementation.

A disadvantage of this approach is that the interface must be carefully resolved around every reinforcement bar. For large structures, this results in sizeable and computationally expensive models, which limits the aforementioned modelling approach to small structures or even parts/regions of the structure. One possibility to reduce the computational effort is to use multiscale modelling methods. In brief, these methods allow to obtain fine resolution results in large-scale models at a fraction of the time it would take to analyse a fully resolved structure. There are many different multiscale methods [10, 11, 12] suitable for this task. Many of them have already been studied and used in modelling of plain concrete [10, 2, $13,14,15,16,17]$ and even reinforced concrete $[18,19,20,21,22]$. One method that shows potential is the $\mathrm{FE}^{2}$ method [23, 24], which couples the scales in a nested way, i.e., the macroscopic response is obtained by computational homogenisation performed on the fine-scale Representative Volume Elements (RVEs), cf. Figure 1. Even though $\mathrm{FE}^{2}$ is computationally expensive, is well suited for parallel computing. Recently, this method has been used for modelling reinforced concrete structures [21, 22], and several further developments enabling the treatment of strain localisation have been made [25, 26, 27, 28].

However, only a few works considered detailed bond-slip modelling with multiscale methods [29, 30, 22]. In recent works by the authors [21, 22], a two-scale model of reinforced concrete was developed and further enriched by a novel effective reinforcement slip macroscopic variable. The enrichment resulted in a localised effective strain field at the macroscale. However, the effective slip variable and its gradient were imposed only on the RVE boundary (Dirichlet boundary conditions), and it was shown that the physical interpretation and effective response of the unit cells were RVE-size dependent, which is undesirable.

In this work, the aforementioned deficiency of the two-scale model is addressed, and the effective reinforcement slip and its gradient are prescribed on the RVE in a volume averaged sense. This weak enforcement of macroscopic variables is performed using Lagrange multipliers, which correspond to the effective work conjugates.

The remainder of the paper is structured as follows: The single-scale problem is briefly formulated in Section 2. In Section 3 the two-scale problem is extensively treated, the volumetric definition of reinforcement slip is proposed, and the corresponding large-scale 


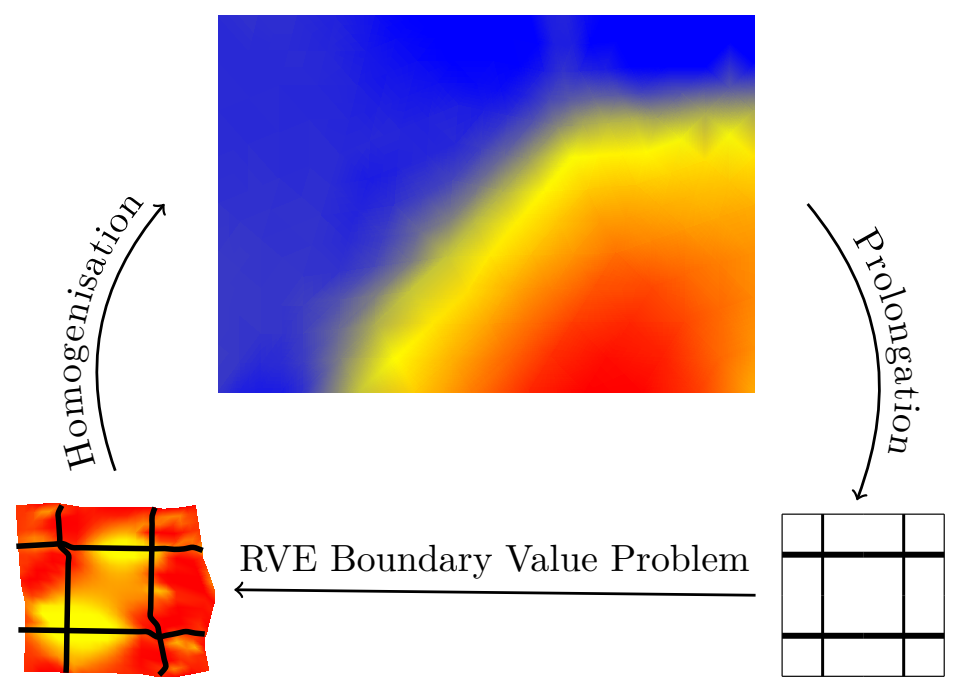

Figure 1: Illustration of the $\mathrm{FE}^{2}$ method.

and subscale problems are derived. The physical meaning of the Lagrange multipliers and their relation to effective work conjugates is outlined. In Section 4, the subscale response of a reinforced concrete RVE at reinforcement pull-through is presented. Section 5 discusses the use of the novel boundary condition in a two-scale analysis of a reinforced deep beam. The paper is concluded with Section 6, which contains some final remarks and an outlook to future work.

\section{Single-scale formulation}

In this section, the single-scale boundary value problem for a general two-dimensional reinforced concrete structure is briefly outlined. The reader is kindly referred to [21] for a more detailed derivation. We consider a reinforced concrete structure occupying the problem domain, $\Omega$, which comprises the concrete part, $\Omega_{\mathrm{c}}$, and the reinforcement part, $\Gamma_{\mathrm{int}}$, schematically depicted in Figure 2. The external boundary is split into the essential $\left(\Gamma_{\mathrm{u}}\right)$ and natural $\left(\Gamma_{\mathrm{t}}\right)$ parts, with either displacement or traction defined, respectively.

Denoting the thickness of the concrete $t_{\mathrm{c}}$, the body force and stress in the concrete $\boldsymbol{b}$ and $\boldsymbol{\sigma}_{\mathrm{c}}$, respectively, we can state the strong form of momentum equilibrium:

$$
\begin{aligned}
-\left(t_{\mathrm{c}} \boldsymbol{\sigma}_{\mathrm{c}}\right) \cdot \boldsymbol{\nabla} & =t_{\mathrm{c}} \boldsymbol{b} & & \text { in } \Omega_{\mathrm{c}}, \\
\boldsymbol{u} & =\boldsymbol{u}_{\mathrm{p}} & & \text { on } \Gamma_{\mathrm{u}}, \\
\boldsymbol{t}:=\boldsymbol{\sigma}_{\mathrm{c}} \cdot \boldsymbol{n} & =\hat{\boldsymbol{t}} & & \text { on } \Gamma_{\mathrm{t}} .
\end{aligned}
$$

For the reinforcement, both longitudinal and transversal action is considered, i.e., both load along and across rebars are allowed. Even though for most applications within modelling of reinforced concrete structures, it would be enough to consider only the longitudinal stiffness of the reinforcement, the transversal stiffness might prove important when modelling shear 


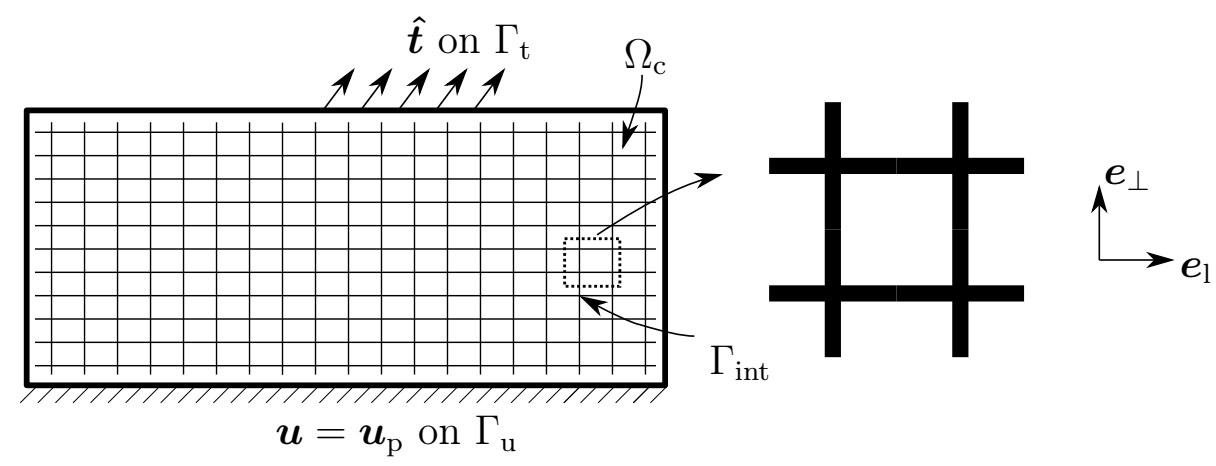

Figure 2: A two-dimensional reinforced concrete structure. For each reinforcement bar, longitudinal and transverse unit vectors $\boldsymbol{e}_{1}$ and $\boldsymbol{e}_{\perp}$ are defined.

failure and shear cracking, where the reinforcement bars cross the inclined crack faces. Thus, the proposed formulation maintains generality, and it can easily be simplified to include only longitudinal action of the reinforcement, if needed. In the model, the normal force, $N_{\mathrm{s}}$, is linked to the bond stress, $t_{\Gamma}$, which is distributed along the perimeter of the bar, $S_{\mathrm{s}}$. Subsequently, we shall assume $S_{\mathrm{s}}$ to be constant along each individual bar, whereas each bar may be of different dimension. Similarly, the bending moment, $M_{\mathrm{s}}$ can be expressed a function of the transverse load, $\lambda$. It is assumed, that there are no external forces (and moments) at the ends of the bars. Hence, the strong form of equilibrium for the reinforcement can be expressed as:

$$
\begin{aligned}
-\frac{\partial N_{\mathrm{s}}}{\partial l}+S_{\mathrm{s}} t_{\Gamma}=0 & \text { in } \Gamma_{\mathrm{int}}, \\
-\frac{\partial^{2} M_{\mathrm{s}}}{\partial l^{2}}+\lambda=0 & \text { in } \Gamma_{\mathrm{int}} \\
N_{\mathrm{s}}=0, T_{\mathrm{s}}=0, M_{\mathrm{s}}=0 & \text { on } \partial \Gamma_{\mathrm{int}} .
\end{aligned}
$$

Considering the steel-concrete interface, for which all the acting forces are schematically represented in Figure 3, we can state the equilibrium condition as

$$
\left[t_{\mathrm{c}}^{+} \boldsymbol{\sigma}_{\mathrm{c}}^{+}\right] \cdot \boldsymbol{e}_{\perp}-\left[t_{\mathrm{c}}^{-} \boldsymbol{\sigma}_{\mathrm{c}}^{-}\right] \cdot \boldsymbol{e}_{\perp}+\lambda \boldsymbol{e}_{\perp}+S_{\mathrm{s}} t_{\Gamma} \boldsymbol{e}_{1}=\mathbf{0} .
$$

Here, the two directions represented by the unit vectors $\boldsymbol{e}_{1}$ and $\boldsymbol{e}_{\perp}$, are introduced. These two vectors can be defined separately for each reinforcement bar comprising the domain $\Gamma_{\text {int }}$. It is noteworthy, that these directions may vary throughout the structure. Moreover, even though only orthogonal reinforcement layouts were studied, it is noted that reinforcement orthogonality is not required for the single-scale formulation to maintain validity.

Next, we introduce the displacement fields $\boldsymbol{u}_{\mathrm{s}}$ and $\boldsymbol{u}_{\mathrm{c}}$ pertinent to steel and concrete, respectively. Along the interface $\Gamma_{\text {int }}$ they can be decomposed into the longitudinal and transversal parts, i.e.,

$$
\begin{aligned}
& \boldsymbol{u}_{\mathrm{s}}=u_{\mathrm{s}, \mathrm{l}} \boldsymbol{e}_{\mathrm{l}}+u_{\mathrm{s}, \perp} \boldsymbol{e}_{\perp}, \\
& \boldsymbol{u}_{\mathrm{c}}=u_{\mathrm{c}, \mathrm{l}} \boldsymbol{e}_{\mathrm{l}}+u_{\mathrm{c}, \perp} \boldsymbol{e}_{\perp} .
\end{aligned}
$$




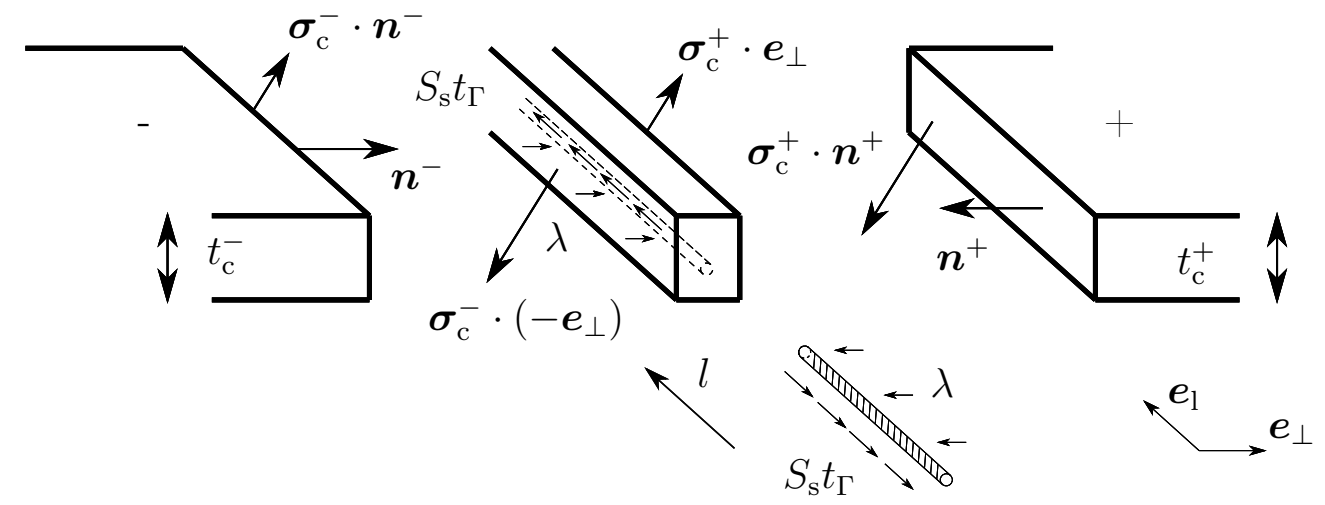

Figure 3: Steel-concrete interface. Boundary forces on the rebar cut out and concrete boundary tractions omitted.

Even though reinforcement slip (difference in the longitudinal deformation of steel and concrete) is allowed, it is assumed that the contact deformation in the transverse direction is negligible. Thus, the following interface constraint is adopted:

$$
u_{\mathrm{s}, \perp}-u_{\mathrm{c}, \perp}=0 \quad \text { in } \Gamma_{\mathrm{int}} .
$$

Here, only implicit definitions of constitutive relations are considered, to maintain generality. In particular, this pertains to the relations between stress and strain in concrete, longitudinal and transversal displacement and the normal forces/bending moments in the steel, and the relation between slip and bond stress in the interface. The strong forms are transformed into weak forms by multiplying the pertinent equations by suitable test functions and integrating over the domain. Moreover, we neglect the body forces in (1) and use the interface equilibrium (3). Hence, the single-scale quasi-static problem can now be defined in the variational form as: Find $\boldsymbol{u}_{\mathrm{c}}, u_{\mathrm{s}, \mathrm{l}}, u_{\mathrm{s}, \perp}, \lambda \in \mathbb{U}_{\mathrm{c}} \times \mathbb{U}_{\mathrm{s}, 1} \times \mathbb{U}_{\mathrm{s}, \perp} \times \mathbb{L}$ such that

$$
\begin{array}{rlrl}
a_{\mathrm{c}}\left(\boldsymbol{u}_{\mathrm{c}} ; \delta \boldsymbol{u}_{\mathrm{c}}\right)-b\left(u_{\mathrm{s}, 1}-\boldsymbol{e}_{\mathrm{l}} \cdot \boldsymbol{u}_{\mathrm{c}} ; \boldsymbol{e}_{1} \cdot \delta \boldsymbol{u}_{\mathrm{c}}\right)-c\left(\lambda ; \boldsymbol{e}_{\perp} \cdot \delta \boldsymbol{u}_{\mathrm{c}}\right) & =l_{\mathrm{c}}\left(\delta \boldsymbol{u}_{\mathrm{c}}\right) & \forall \delta \boldsymbol{u}_{\mathrm{c}} \in \mathbb{U}_{\mathrm{c}}^{0}, \\
a_{1}\left(u_{\mathrm{s}, 1}, u_{\mathrm{s}, \perp} ; \delta u_{\mathrm{s}, 1}\right)+b\left(u_{\mathrm{s}, 1}-\boldsymbol{e}_{\mathrm{l}} \cdot \boldsymbol{u}_{\mathrm{c}} ; \delta u_{\mathrm{s}, 1}\right) & =0 & \forall \delta u_{\mathrm{s}, 1} \in \mathbb{U}_{\mathrm{s}, 1}, \\
a_{\mathrm{b}}\left(u_{\mathrm{s}, 1}, u_{\mathrm{s}, \perp} ; \delta u_{\mathrm{s}, \perp}\right)+c\left(\lambda ; \delta u_{\mathrm{s}, \perp}\right) & =0 & \forall \delta u_{\mathrm{s}, \perp} \in \mathbb{U}_{\mathrm{s}, \perp}, \\
c\left(\delta \lambda ; u_{\mathrm{s}, \perp}-\boldsymbol{e}_{\perp} \cdot \boldsymbol{u}_{\mathrm{c}}\right)=0 & \forall \delta \lambda \in \mathbb{L},
\end{array}
$$

for suitable trial spaces $\mathbb{U}_{\mathrm{c}}, \mathbb{U}_{\mathrm{s}, \mathrm{l}}, \mathbb{U}_{\mathrm{s}, \perp}, \mathbb{L}$ :

$$
\begin{aligned}
\mathbb{U}_{\mathrm{c}} & =\left\{\boldsymbol{u}(\boldsymbol{x}): \Omega \mapsto \mathbb{R}^{2}, \int_{\Omega_{\mathrm{c}}} \boldsymbol{u}^{2}+[\boldsymbol{u} \otimes \nabla]^{2} \mathrm{~d} \Omega<\infty, \boldsymbol{u}=\boldsymbol{u}_{\mathrm{p}} \text { on } \Gamma_{\mathrm{u}}\right\}, \\
\mathbb{U}_{\mathrm{s}, \mathrm{l}} & =\left\{v(l): \Gamma_{\mathrm{int}} \mapsto \mathbb{R}, \int_{\Gamma_{\mathrm{int}}} v^{2}+\left(\frac{\partial v}{\partial l}\right)^{2} \mathrm{~d} \Gamma<\infty\right\}, \\
\mathbb{U}_{\mathrm{s}, \perp} & =\left\{v(l): \Gamma_{\mathrm{int}} \mapsto \mathbb{R}, \int_{\Gamma_{\mathrm{int}}} v^{2}+\left(\frac{\partial v}{\partial l}\right)^{2}+\left(\frac{\partial^{2} v}{\partial l^{2}}\right)^{2} \mathrm{~d} \Gamma<\infty\right\}, \\
\mathbb{L} & =\left\{\lambda(l): \Gamma_{\mathrm{int}} \mapsto \mathbb{R}, \int_{\Gamma_{\mathrm{int}}} \lambda^{2} \mathrm{~d} \Gamma<\infty\right\},
\end{aligned}
$$


and the test space $\mathbb{U}_{\mathrm{c}}^{0}$ :

$$
\mathbb{U}_{\mathrm{c}}^{0}=\left\{\boldsymbol{u}(\boldsymbol{x}): \Omega \mapsto \mathbb{R}^{2}, \int_{\Omega_{\mathrm{c}}} \boldsymbol{u}^{2}+[\boldsymbol{u} \otimes \nabla]^{2} \mathrm{~d} \Omega<\infty, \boldsymbol{u}=\mathbf{0} \text { on } \Gamma_{\mathrm{u}}\right\} .
$$

The coupling terms in the system (7)-(10) are defined as:

$$
\begin{aligned}
b(v ; w) & :=\int_{\Gamma_{\mathrm{int}}} S_{\mathrm{s}} t_{\Gamma}(v) w \mathrm{~d} \Gamma, \\
c(\lambda ; v) & :=\int_{\Gamma_{\mathrm{int}}} \lambda v \mathrm{~d} \Gamma .
\end{aligned}
$$

The following forms are introduced pertinent to:

(i) Concrete:

$$
\begin{aligned}
a_{\mathrm{c}}\left(\boldsymbol{u}_{\mathrm{c}} ; \delta \boldsymbol{u}_{\mathrm{c}}\right) & :=\int_{\Omega_{\mathrm{c}}} t_{\mathrm{c}} \boldsymbol{\sigma}_{\mathrm{c}}\left(\boldsymbol{\varepsilon}\left[\boldsymbol{u}_{\mathrm{c}}\right]\right):\left[\delta \boldsymbol{u}_{\mathrm{c}} \otimes \nabla\right] \mathrm{d} \Omega, \\
l_{\mathrm{c}}\left(\delta \boldsymbol{u}_{\mathrm{c}}\right) & :=\int_{\Gamma_{\mathrm{ext}}} t_{\mathrm{c}} \hat{\boldsymbol{t}} \cdot \delta \boldsymbol{u}_{\mathrm{c}} \mathrm{d} \Gamma .
\end{aligned}
$$

(ii) Bar action of the rebars:

$$
a_{\mathrm{l}}\left(u_{\mathrm{s}, 1}, u_{\mathrm{s}, \perp} ; \delta u_{\mathrm{s}, 1}\right):=\int_{\Gamma_{\mathrm{int}}} N_{\mathrm{s}}\left(\frac{\partial u_{\mathrm{s}, 1}}{\partial l}, \frac{\partial^{2} u_{\mathrm{s}, \perp}}{\partial l^{2}}\right) \frac{\partial \delta u_{\mathrm{s}, 1}}{\partial l} \mathrm{~d} \Gamma
$$

(iii) Beam action of the rebars:

$$
a_{\mathrm{b}}\left(u_{\mathrm{s}, 1}, u_{\mathrm{s}, \perp} ; \delta u_{\mathrm{s}, \perp}\right):=-\int_{\Gamma_{\mathrm{int}}} M_{\mathrm{s}}\left(\frac{\partial u_{\mathrm{s}, 1}}{\partial l}, \frac{\partial^{2} u_{\mathrm{s}, \perp}}{\partial l^{2}}\right) \frac{\partial^{2} \delta u_{\mathrm{s}, \perp}}{\partial l^{2}} \mathrm{~d} \Gamma .
$$

\section{Two-scale formulation}

In view of the fact, that the physical scale of the homogeneous concrete and distinct reinforcement bars is clearly macroscopic, the terms macroscale and microscale, commonly used in the literature are, in this paper, substituted (or used interchangeably) with the terms large-scale and subscale.

\subsection{Variationally Consistent Homogenisation}

In this paper, we use the so-called Variationally Consistent Homogenisation [31] technique to separate the scales. In short, the unknown fields $\boldsymbol{u}_{\mathrm{c}}$ and $\boldsymbol{u}_{\mathrm{s}}$ are separated into smooth (large-scale) and fluctuating (subscale) parts:

$$
\begin{aligned}
& \boldsymbol{u}_{\mathrm{c}}=\boldsymbol{u}_{\mathrm{c}}^{M}+\boldsymbol{u}_{\mathrm{c}}^{s}, \\
& \boldsymbol{u}_{\mathrm{s}}=\boldsymbol{u}_{\mathrm{s}}^{M}+\boldsymbol{u}_{\mathrm{s}}^{s},
\end{aligned}
$$


where the superscripts $M$ and $s$ represent large-scale and subscale components, respectively. The decomposition of fields pertaining to longitudinal and transversal deformation of reinforcement is simply obtained by projecting the field $\boldsymbol{u}_{\mathrm{s}}$ on the corresponding direction, i.e.,

$$
\begin{gathered}
u_{\mathrm{s}, 1}=\boldsymbol{e}_{\mathrm{l}} \cdot \boldsymbol{u}_{\mathrm{s}}=u_{\mathrm{s}, 1}^{M}+u_{\mathrm{s}, 1}^{s}, \\
u_{\mathrm{s}, \perp}=\boldsymbol{e}_{\perp} \cdot \boldsymbol{u}_{\mathrm{s}}=u_{\mathrm{s}, \perp}^{M}+u_{\mathrm{s}, \perp}^{s} .
\end{gathered}
$$

The natural ramification of this split is that each point in the problem domain is reflected at two levels, and thus two different problems can be constructed. At the first level, the largescale problem is defined in terms of globally "smooth" fields, different from the unknown fields defined in (17) and (18). At the second level, the substructure of the material can be represented by the so-called Representative Volume Element (RVE) occupying the region $\Omega_{\square}$ in the vicinity of the considered point. In the two-scale model setting, the local field is replaced by the homogenised field, i.e. at each location $\overline{\boldsymbol{x}} \in \Omega$ the field is approximated by the volume average on $\Omega_{\square}(\overline{\boldsymbol{x}})$. In particular, for given functions $f_{\Omega}$ and $f_{\Gamma}$ defined on $\Omega_{\mathrm{c}}$ and $\Gamma_{\text {int }}$, respectively, we have

$$
\int_{\Omega_{\mathrm{c}}} f_{\Omega} \mathrm{d} \Omega+\int_{\Gamma_{\mathrm{int}}} f_{\Gamma} \mathrm{d} \Gamma \mapsto \int_{\Omega} f_{\square} \mathrm{d} \Omega
$$

where the subscale average $f_{\square}$ is defined as:

$$
f_{\square}=\frac{1}{\left|\Omega_{\square}\right|}\left\{\int_{\Omega_{\square, \mathrm{c}}} f_{\Omega} \mathrm{d} \Omega+\int_{\Gamma_{\square, \mathrm{int}}} f_{\Gamma} \mathrm{d} \Gamma\right\} .
$$

Using the averages, the single-scale problem defined in (7)-(10) can be rewritten as

$$
\begin{gathered}
\int_{\Omega}\left[a_{\square, \mathrm{c}}\left(\boldsymbol{u}_{\mathrm{c}} ; \delta \boldsymbol{u}_{\mathrm{c}}\right)+a_{\square, 1}\left(u_{\mathrm{s}, 1}, u_{\mathrm{s}, \perp} ; \delta u_{\mathrm{s}, 1}\right)+a_{\square, \mathrm{b}}\left(u_{\mathrm{s}, \mathrm{l}}, u_{\mathrm{s}, \perp} ; \delta u_{\mathrm{s}, \perp}\right)+c_{\square}\left(\lambda ; \delta u_{\mathrm{s}, \perp}-\boldsymbol{e}_{\perp} \cdot \delta \boldsymbol{u}_{\mathrm{c}}\right)\right. \\
\left.+b_{\square}\left(u_{\mathrm{s}, 1}-\boldsymbol{e}_{\mathrm{l}} \cdot \boldsymbol{u}_{\mathrm{c}} ; \delta u_{\mathrm{s}, 1}-\boldsymbol{e}_{\mathrm{l}} \cdot \delta \boldsymbol{u}_{\mathrm{c}}\right)+c_{\square}\left(\delta \lambda ; u_{\mathrm{s}, \perp}-\boldsymbol{e}_{\perp} \cdot \boldsymbol{u}_{\mathrm{c}}\right)\right] \mathrm{d} \Omega=l_{\mathrm{c}}\left(\delta \boldsymbol{u}_{\mathrm{c}}\right),
\end{gathered}
$$

where we introduced the RVE-forms

$$
\begin{aligned}
a_{\square, \mathrm{c}}\left(\boldsymbol{u}_{\mathrm{c}} ; \delta \boldsymbol{u}_{\mathrm{c}}\right) & :=\frac{1}{\left|\Omega_{\square}\right|} \int_{\Omega_{\square, \mathrm{c}}} t_{\mathrm{c}} \boldsymbol{\sigma}_{\mathrm{c}}:\left[\delta \boldsymbol{u}_{\mathrm{c}} \otimes \nabla\right] \mathrm{d} \Omega, \\
b_{\square}(v ; w) & :=\frac{1}{\left|\Omega_{\square}\right|} \int_{\Gamma_{\square, \mathrm{int}}} S_{\mathrm{s}} t_{\Gamma}(v) w \mathrm{~d} \Gamma, \\
c_{\square}(\lambda ; w) & :=\frac{1}{\left|\Omega_{\square}\right|} \int_{\Gamma_{\square, \mathrm{int}}} \lambda v \mathrm{~d} \Gamma, \\
a_{\square, 1}\left(u_{\mathrm{s}, 1}, u_{\mathrm{s}, \perp} ; \delta u_{\mathrm{s}, 1}\right) & :=\frac{1}{\left|\Omega_{\square}\right|} \int_{\Gamma_{\square, \mathrm{int}}} N_{\mathrm{s}} \frac{\partial \delta u_{\mathrm{s}, 1}}{\partial l} \mathrm{~d} \Gamma, \\
a_{\square, \mathrm{b}}\left(u_{\mathrm{s}, 1}, u_{\mathrm{s}, \perp} ; \delta u_{\mathrm{s}, \perp}\right) & :=\frac{1}{\left|\Omega_{\square}\right|} \int_{\Gamma_{\square, \mathrm{int}}}-M_{\mathrm{s}} \frac{\partial^{2} \delta u_{\mathrm{s}, \perp}}{\partial l^{2}} \mathrm{~d} \Gamma .
\end{aligned}
$$


Since in practice, numerical integration is performed at Gauss points, it suffices to consider the subscale problems at these locations, while solving the large-scale problem. The "smooth" large-scale field is imposed on the RVEs with the help of Taylor expansion. In this paper, first-order (expansion up to linear term) computational homogenisation is used, cf. [32].

\subsection{Volumetric definition of the effective slip}

In contrast to the classical structural formulation [21], we introduce two independent large-scale fields, the effective displacement $\overline{\boldsymbol{u}}$ and the effective reinforcement slip $\overline{\boldsymbol{s}}$. Following the procedure proposed in [31], we aim at prolonging the large-scale components of the resolved fields $(17,18)$ from the effective large-scale fields, i.e., we want to establish an operator $\mathcal{A}$ such that $\left(\boldsymbol{u}_{\mathrm{c}}^{M}, \boldsymbol{u}_{\mathrm{s}}^{M}\right)=\mathcal{A}(\overline{\boldsymbol{u}}, \overline{\boldsymbol{s}})$. More specifically, we consider a Taylor series expansion of the large-scale fields inside $\Omega_{\square}$, centered around $\overline{\boldsymbol{x}}=\frac{1}{\left|\Omega_{\square}\right|} \int_{\Omega_{\square}} \boldsymbol{x} \mathrm{d} \Omega$, as follows:

$$
\begin{aligned}
\boldsymbol{u}_{\mathrm{c}}^{M}(\overline{\boldsymbol{x}}, \boldsymbol{x}) & =\overline{\boldsymbol{u}}(\overline{\boldsymbol{x}})+\overline{\boldsymbol{\varepsilon}}(\overline{\boldsymbol{x}}) \cdot[\boldsymbol{x}-\overline{\boldsymbol{x}}] \\
u_{\mathrm{s}, 1}^{M}(\overline{\boldsymbol{x}}, \boldsymbol{x}) & =\boldsymbol{e}_{\mathrm{l}} \cdot \boldsymbol{u}_{\mathrm{c}}^{M}(\overline{\boldsymbol{x}}, \boldsymbol{x})+\boldsymbol{e}_{1} \cdot \overline{\boldsymbol{s}}(\overline{\boldsymbol{x}})+\boldsymbol{e}_{1} \cdot \overline{\boldsymbol{g}}_{\mathrm{d}}(\overline{\boldsymbol{x}}) \cdot[\boldsymbol{x}-\overline{\boldsymbol{x}}] \\
& =\boldsymbol{e}_{\mathrm{l}} \cdot[\overline{\boldsymbol{u}}(\overline{\boldsymbol{x}})+\overline{\boldsymbol{s}}(\overline{\boldsymbol{x}})]+\boldsymbol{e}_{\mathrm{l}} \cdot\left[\overline{\boldsymbol{\varepsilon}}(\overline{\boldsymbol{x}})+\overline{\boldsymbol{g}}_{\mathrm{d}}(\overline{\boldsymbol{x}})\right] \cdot[\boldsymbol{x}-\overline{\boldsymbol{x}}], \\
u_{\mathrm{s}, \perp}^{M}(\overline{\boldsymbol{x}}, \boldsymbol{x}) & =\boldsymbol{e}_{\perp} \cdot \boldsymbol{u}_{\mathrm{c}}^{M}(\overline{\boldsymbol{x}}, \boldsymbol{x})=\boldsymbol{e}_{\perp} \cdot \overline{\boldsymbol{u}}(\overline{\boldsymbol{x}})+\boldsymbol{e}_{\perp} \cdot \overline{\boldsymbol{\varepsilon}}(\overline{\boldsymbol{x}}) \cdot[\boldsymbol{x}-\overline{\boldsymbol{x}}],
\end{aligned}
$$

where the large-scale strain and diagonal gradient of slip are defined as

$$
\begin{aligned}
\overline{\boldsymbol{\varepsilon}}: & =[\overline{\boldsymbol{u}} \otimes \boldsymbol{\nabla}]^{\mathrm{sym}}, \\
\overline{\boldsymbol{g}}_{\mathrm{d}}: & =\mathbb{I}_{\mathrm{d}}:[\overline{\boldsymbol{s}} \otimes \boldsymbol{\nabla}],
\end{aligned}
$$

respectively. Here, $\mathbb{I}_{\mathrm{d}}=\sum_{i=1}^{2} \boldsymbol{e}_{\mathrm{l}, \mathrm{i}} \otimes \boldsymbol{e}_{\mathrm{l}, \mathrm{i}} \otimes \boldsymbol{e}_{\mathrm{l}, \mathrm{i}} \otimes \boldsymbol{e}_{\mathrm{l}, \mathrm{i}}$ extracts the diagonal part of $[\overline{\boldsymbol{s}} \otimes \boldsymbol{\nabla}]$ assuming only two orthogonal reinforcement directions $\left(\boldsymbol{e}_{1,1}, \boldsymbol{e}_{1,2}\right)$ inside the RVE. As a result from this modelling assumption, only large-scale gradients along a reinforcement bar will be accounted for at the large-scale. The fact that it is only $\bar{\varepsilon}$ and not the entire gradient $[\overline{\boldsymbol{u}} \otimes \nabla]$ that is imposed to $\boldsymbol{u}_{\mathrm{c}}^{M}, \boldsymbol{u}_{\mathrm{s}}^{M}$ follows from the invariance to rigid body motion, cf. e.g. [21].

The homogenisation of fields, which in fact defines the large-scale fields, can be expressed on an RVE by an operator $\mathcal{A}^{*}$ such that $(\overline{\boldsymbol{u}}, \overline{\boldsymbol{s}})=\mathcal{A}^{*}\left(\boldsymbol{u}_{\mathrm{c}}, \boldsymbol{u}_{\mathrm{s}}\right)$. Here $\left(\boldsymbol{u}_{\mathrm{c}}, \boldsymbol{u}_{\mathrm{s}}\right)$ denotes the parts of the fields that are introduced in the prolongation (29)-(31). Hence, we need to introduce measures on $\Omega_{\square}$ that define

$$
\begin{array}{cr}
\overline{\boldsymbol{u}}=\overline{\boldsymbol{u}}_{\square}\left(\boldsymbol{u}_{\mathrm{c}}, \boldsymbol{u}_{\mathrm{s}}\right), & \overline{\boldsymbol{\varepsilon}}=\overline{\boldsymbol{\varepsilon}}_{\square}\left(\boldsymbol{u}_{\mathrm{c}}, \boldsymbol{u}_{\mathrm{s}}\right), \\
\overline{\boldsymbol{s}}=\overline{\boldsymbol{s}}_{\square}\left(\boldsymbol{u}_{\mathrm{c}}, \boldsymbol{u}_{\mathrm{s}}\right), & \overline{\boldsymbol{g}}_{\mathrm{d}}=\overline{\boldsymbol{g}}_{\mathrm{d}, \square}\left(\boldsymbol{u}_{\mathrm{c}}, \boldsymbol{u}_{\mathrm{s}}\right) .
\end{array}
$$

In order to guarantee a well-defined scale-coupling, we shall require that $\mathcal{A}^{*} \mathcal{A}=\mathbb{I}$, i.e.,

$$
\begin{array}{rlrl}
\overline{\boldsymbol{u}}_{\square}\left(\boldsymbol{u}_{\mathrm{c}}^{M}, \boldsymbol{u}_{\mathrm{s}}^{M}\right) & =\overline{\boldsymbol{u}}, & \bar{\varepsilon}_{\square}\left(\boldsymbol{u}_{\mathrm{c}}^{M}, \boldsymbol{u}_{\mathrm{s}}^{M}\right)=\overline{\boldsymbol{\varepsilon}}, \\
\overline{\boldsymbol{s}}_{\square}\left(\boldsymbol{u}_{\mathrm{c}}^{M}, \boldsymbol{u}_{\mathrm{s}}^{M}\right)=\overline{\boldsymbol{s}}, & \overline{\boldsymbol{g}}_{\mathrm{d}, \square}\left(\boldsymbol{u}_{\mathrm{c}}^{M}, \boldsymbol{u}_{\mathrm{s}}^{M}\right)=\overline{\boldsymbol{g}}_{\mathrm{d}} .
\end{array}
$$


for the prolongation given in eqs. (29) and (31). Together with the restriction of the subscale fields $\mathcal{A}^{*}\left(\boldsymbol{u}_{\mathrm{c}}^{s}, \boldsymbol{u}_{\mathrm{s}}^{s}\right)=\mathbf{0}$, the requirements in (36)-(37) certify that the large-scale fields is a unique parameterisation of the (total) resolved field $\left(\boldsymbol{u}_{\mathrm{c}}, \boldsymbol{u}_{\mathrm{s}}\right)$.

Considering the displacement of concrete, the definitions of the large-scale displacement and strain are standard, see e.g. [33],

$$
\begin{aligned}
\overline{\boldsymbol{u}}_{\square}\left(\boldsymbol{u}_{\mathrm{c}}, \boldsymbol{u}_{\mathrm{s}}\right)=\overline{\boldsymbol{u}}_{\square, \mathrm{c}}\left(\boldsymbol{u}_{\mathrm{c}}\right):=\frac{1}{\left|\Omega_{\square}\right|} \int_{\Omega_{\square}} \boldsymbol{u}_{\mathrm{c}} \mathrm{d} \Omega, \\
\overline{\boldsymbol{\varepsilon}}_{\square}\left(\boldsymbol{u}_{\mathrm{c}}, \boldsymbol{u}_{\mathrm{s}}\right)=\overline{\boldsymbol{\varepsilon}}_{\square, \mathrm{c}}\left(\boldsymbol{u}_{\mathrm{c}}\right):=\frac{1}{\left|\Omega_{\square}\right|} \int_{\Omega_{\square}}\left[\boldsymbol{u}_{\mathrm{c}} \otimes \nabla\right]^{\mathrm{sym}} \mathrm{d} \Omega
\end{aligned}
$$

and do satisfy the corresponding criteria in (36)-(37). In particular, we note that the expressions are independent of $\boldsymbol{u}_{\mathrm{s}}$, and thus $\overline{\boldsymbol{s}}$ and $\overline{\boldsymbol{g}}_{\mathrm{d}}$ when considering $\boldsymbol{u}_{\mathrm{c}}^{M}$.

As to the large-scale reinforcement slip, we propose to define it as the difference between large-scale averaged displacements,

$$
\overline{\boldsymbol{s}}_{\square}\left(\boldsymbol{u}_{\mathrm{c}}, \boldsymbol{u}_{\mathrm{s}}\right)=\overline{\boldsymbol{u}}_{\square, \mathrm{s}}\left(\boldsymbol{u}_{\mathrm{s}}\right)-\overline{\boldsymbol{u}}_{\square, \mathrm{c}}\left(\boldsymbol{u}_{\mathrm{c}}\right) .
$$

Here, the concrete displacement is exactly that of the overall displacement, as introduced in Equation (38), and the steel displacement is measured along each reinforcement bar as

$$
\overline{\boldsymbol{u}}_{\square, \mathrm{s}}\left(\boldsymbol{u}_{\mathrm{s}}\right)=\frac{1}{\left|\Omega_{\square}\right|} \int_{\Gamma_{\square, \mathrm{int}}} S_{\mathrm{s}} u_{\mathrm{s}, \mathrm{l}} \boldsymbol{e}_{\mathrm{l}} \mathrm{d} \Gamma \cdot \boldsymbol{C}_{\mathrm{s}} .
$$

The ansatz in Equation (41) contains a scaling tensor $\boldsymbol{C}_{\mathrm{s}}$ that has to be determined in order to satisfy the constraints in (36)-(37). It can be shown that setting

$$
\boldsymbol{C}_{\mathrm{s}}=\left|\Omega_{\square}\right|\left[\int_{\Gamma_{\square, \mathrm{int}}} S_{\mathrm{s}} \boldsymbol{e}_{\mathrm{l}} \otimes \boldsymbol{e}_{\mathrm{l}} \mathrm{d} \Gamma\right]^{-1} .
$$

satisfies the mentioned criteria. For details concerning derivation, the reader is referred to Appendix A. For the diagonal gradient of slip, we propose the definition

$$
\overline{\boldsymbol{g}}_{\square}\left(\boldsymbol{u}_{\mathrm{c}}, \boldsymbol{u}_{\mathrm{s}}\right)=\overline{\boldsymbol{g}}_{\mathrm{d}, \square, \mathrm{s}}\left(\boldsymbol{u}_{\mathrm{s}}\right)-\overline{\boldsymbol{g}}_{\mathrm{d}, \square, \mathrm{c}}\left(\boldsymbol{u}_{\mathrm{c}}\right),
$$

with the expressions

$$
\begin{aligned}
& \overline{\boldsymbol{g}}_{\mathrm{d}, \square, \mathrm{c}}\left(\boldsymbol{u}_{\mathrm{c}}\right)=\mathbb{I}_{\mathrm{d}}: \overline{\boldsymbol{\varepsilon}}_{\square, \mathrm{c}}\left(\boldsymbol{u}_{\mathrm{c}}\right)=\frac{1}{\left|\Omega_{\square}\right|} \int_{\Omega_{\square}}\left[\boldsymbol{u}_{\mathrm{c}} \otimes \boldsymbol{\nabla}\right]^{\mathrm{sym}} \mathrm{d} \Omega: \mathbb{I}_{\mathrm{d}}, \\
& \overline{\boldsymbol{g}}_{\mathrm{d}, \square, \mathrm{s}}\left(\boldsymbol{u}_{\mathrm{s}}\right)=\frac{1}{\left|\Omega_{\square}\right|} \int_{\Gamma_{\square, \mathrm{int}}} S_{\mathrm{s}} \frac{\partial u_{\mathrm{s}, \mathrm{l}}}{\partial l} \boldsymbol{e}_{\mathrm{l}} \otimes \boldsymbol{e}_{\mathrm{l}} \mathrm{d} \Gamma \cdot \boldsymbol{C}_{\mathrm{g}} .
\end{aligned}
$$

Analogous to Equation (41), we here introduce a constant tensor for scaling the measure, that has to be determined in order to satisfy the criteria (36)-(37). It can be shown that setting

$$
C_{\mathrm{g}}=C_{\mathrm{s}}
$$


satisfies the mentioned criteria. For the corresponding proof, the reader is referred to Appendix A. Recall that we assume each bar to have a uniform cross section. Hence, we may use integration along each bar inside the RVE to obtain the identity

$$
\overline{\boldsymbol{g}}_{\mathrm{d}, \square, \mathrm{s}}\left(\boldsymbol{u}_{\mathrm{s}}\right)=\left[\left.\frac{1}{\left|\Omega_{\square}\right|} \sum_{i}\left(S_{\mathrm{s}} e_{\mathrm{nl}} u_{\mathrm{s}, \mathrm{l}} \boldsymbol{e}_{\mathrm{l}} \otimes \boldsymbol{e}_{\mathrm{l}}\right)\right|_{\boldsymbol{x}_{i}}\right] \cdot \boldsymbol{C}_{\mathrm{s}} .
$$

Here, the set $\left\{\boldsymbol{x}_{\mathrm{i}}\right\}$ denotes the endpoints of bars, and $e_{\mathrm{nl}}=1$ and -1 at start- and endpoints ${ }^{1}$, respectively.

\subsection{Large-scale problem}

The large-scale problem is defined in terms of the globally "smooth" fields $\overline{\boldsymbol{u}}$ and $\overline{\boldsymbol{s}}$. It can be derived from the variational formulation (23) by restricting the choice of test functions to the macroscopic part coming from the Variational MultiScale (VMS) ansatz, i.e.,

$$
\begin{aligned}
& \int_{\Omega}\left[a_{\square, \mathrm{c}}\left(\bullet ; \delta \boldsymbol{u}_{\mathrm{c}}^{M}\right)+b_{\square}\left(\bullet ; \delta u_{\mathrm{s}, 1}^{M}-\boldsymbol{e}_{1} \cdot \delta \boldsymbol{u}_{\mathrm{c}}^{M}\right)+c_{\square}\left(\bullet ; \delta u_{\mathrm{s}, \perp}^{M}-\boldsymbol{e}_{\perp} \cdot \delta \boldsymbol{u}_{\mathrm{c}}^{M}\right)+a_{\square, 1}\left(\bullet ; \delta u_{\mathrm{s}, 1}^{M}\right)\right. \\
& \left.\quad+a_{\square, \mathrm{b}}\left(\bullet ; \delta u_{\mathrm{s}, \perp}^{M}\right)\right] \mathrm{d} \Omega=l_{\mathrm{c}}\left(\delta \boldsymbol{u}_{\mathrm{c}}^{M}\right) .
\end{aligned}
$$

The local field at the domain boundary $\Gamma_{\text {ext }}$ is assumed to be sufficiently smooth. Hence, $l_{\mathrm{c}}\left(\delta \boldsymbol{u}_{\mathrm{c}}^{M}\right) \approx l_{\mathrm{c}}(\delta \overline{\boldsymbol{u}})$. Employing the prolongation conditions (29)-(31), we have

$$
\int_{\Omega} \overline{\boldsymbol{\sigma}}: \overline{\boldsymbol{\varepsilon}}[\delta \overline{\boldsymbol{u}}]+\overline{\boldsymbol{\tau}}_{\mathrm{b}} \cdot \delta \overline{\boldsymbol{s}}+\overline{\boldsymbol{\sigma}}_{\mathrm{s}}: \overline{\boldsymbol{g}}_{\mathrm{d}}[\delta \overline{\boldsymbol{s}}] \mathrm{d} \Omega=\int_{\Gamma_{\mathrm{ext}}} t_{\mathrm{c}} \hat{\boldsymbol{t}} \cdot \delta \overline{\boldsymbol{u}} \mathrm{d} \Gamma,
$$

where the quantities $\overline{\boldsymbol{\sigma}}, \overline{\boldsymbol{\tau}}_{\mathrm{b}}, \overline{\boldsymbol{\sigma}}_{\mathrm{s}}$ are named the effective stress, effective transfer stress and effective reinforcement stress, and are defined as:

$$
\begin{aligned}
\overline{\boldsymbol{\sigma}} & =\frac{1}{\left|\Omega_{\square}\right|}\left\{\int_{\Omega_{\square, \mathrm{c}}} t_{\mathrm{c}} \boldsymbol{\sigma}_{\mathrm{c}} \mathrm{d} \Omega+\int_{\Gamma_{\square, \mathrm{int}}} N_{\mathrm{s}} \boldsymbol{e}_{\mathrm{l}} \otimes \boldsymbol{e}_{\mathrm{l}} \mathrm{d} \Gamma\right\}, \\
\overline{\boldsymbol{\tau}}_{\mathrm{b}} & =\frac{1}{\left|\Omega_{\square}\right|} \int_{\Gamma_{\square, \mathrm{int}}} S_{\mathrm{s}} t_{\Gamma} \boldsymbol{e}_{\mathrm{l}} \mathrm{d} \Gamma, \\
\overline{\boldsymbol{\sigma}}_{\mathrm{s}} & =\mathbb{I}_{\mathrm{d}}: \frac{1}{\left|\Omega_{\square}\right|} \int_{\Gamma_{\square, \mathrm{int}}} S_{\mathrm{s}} t_{\Gamma} \boldsymbol{e}_{\mathrm{l}} \otimes[\boldsymbol{x}-\overline{\boldsymbol{x}}]+N_{\mathrm{s}} \boldsymbol{e}_{\mathrm{l}} \otimes \boldsymbol{e}_{\mathrm{l}} \mathrm{d} \Gamma .
\end{aligned}
$$

Hence, the large-scale problem is defined as: Find $\overline{\boldsymbol{u}}, \overline{\boldsymbol{s}} \in \overline{\mathbb{U}} \times \overline{\mathbb{S}}$ such that

$$
\begin{array}{cc}
\int_{\Omega} \overline{\boldsymbol{\sigma}}: \overline{\boldsymbol{\varepsilon}}[\delta \overline{\boldsymbol{u}}] \mathrm{d} \Omega=\int_{\Gamma_{\mathrm{ext}}} t_{\mathrm{c}} \hat{\boldsymbol{t}} \cdot \delta \overline{\boldsymbol{u}} \mathrm{d} \Gamma & \forall \delta \overline{\boldsymbol{u}} \in \overline{\mathbb{U}}^{0}, \\
\int_{\Omega} \overline{\boldsymbol{\tau}}_{\mathrm{b}} \cdot \delta \overline{\boldsymbol{s}}+\overline{\boldsymbol{\sigma}}_{\mathrm{s}}: \overline{\boldsymbol{g}}_{\mathrm{d}}[\delta \overline{\boldsymbol{s}}] \mathrm{d} \Omega=0 & \forall \delta \overline{\boldsymbol{s}} \in \overline{\mathbb{S}}
\end{array}
$$

\footnotetext{
${ }^{1}$ Start- and endpoints of the bar are defined through the direction of $\boldsymbol{e}_{1}$.
} 


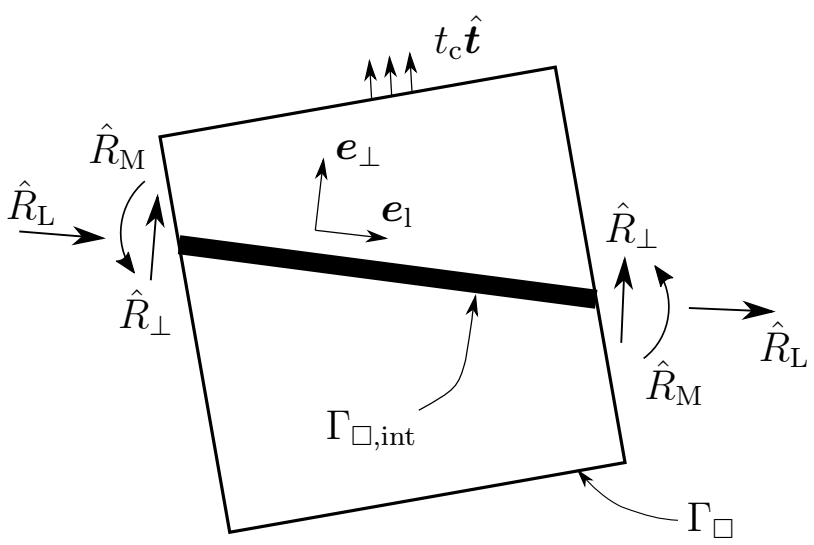

Figure 4: Discrete forces and traction at the boundary $\Gamma_{\square}$ of a unit cell.

with the suitable trial and test spaces

$$
\begin{aligned}
& \overline{\mathbb{U}}=\left\{\boldsymbol{u}(\boldsymbol{x}): \Omega \mapsto \mathbb{R}^{2}, \int_{\Omega} \boldsymbol{u}^{2}+[\boldsymbol{u} \otimes \boldsymbol{\nabla}]^{2} \mathrm{~d} \Omega<\infty, \boldsymbol{u}=\boldsymbol{u}_{\mathrm{p}} \text { on } \Gamma_{\mathrm{u}}\right\}, \\
& \overline{\mathbb{U}}^{0}=\left\{\boldsymbol{u}(\boldsymbol{x}): \Omega \mapsto \mathbb{R}^{2}, \int_{\Omega} \boldsymbol{u}^{2}+[\boldsymbol{u} \otimes \boldsymbol{\nabla}]^{2} \mathrm{~d} \Omega<\infty, \boldsymbol{u}=\mathbf{0} \text { on } \Gamma_{\mathrm{u}}\right\}, \\
& \overline{\mathbb{S}}=\left\{\boldsymbol{s}(\boldsymbol{x}): \Omega \mapsto \mathbb{R}^{2}, \int_{\Omega} \boldsymbol{s}^{2}+[\boldsymbol{s} \otimes \boldsymbol{\nabla}]^{2} \mathrm{~d} \Omega<\infty\right\} .
\end{aligned}
$$

Remark:

We note that the regularity requirements on functions $\overline{\boldsymbol{s}} \in \overline{\mathbb{S}}$ could be relaxed, since it is only $\mathbb{I}_{\mathrm{d}}:[\overline{\boldsymbol{s}} \otimes \boldsymbol{\nabla}]$ that needs to be bounded. However, this detail (as well as the possibility to use discontinuous FE approximation) is not considered further.

\subsection{Subscale problem}

The subscale problem on the RVE follows directly from (23), when restricting the choice of test function to the fluctuation fields, according to the VMS split. However, it is often more feasible from the computational point of view, to solve for the total fields, and not only for the fluctuation parts. Moreover, since the RVE domain $\Omega_{\square}$ is usually much smaller than the problem domain $\Omega$, the assumption of vanishing end forces (and tractions) for the reinforcement and concrete is not valid. Hence, the discrete end forces and boundary tractions terms need to be reflected in the formulation. In such a general case, it is advantageous to state the problem in its canonical form, whereby the physical interpretation of imposed Lagrange multipliers can be easily found. 


\subsubsection{Canonical format}

The subscale solution fields $\boldsymbol{u}_{\mathrm{c}}, u_{\mathrm{s}, \mathrm{l}}, u_{\mathrm{s}, \perp}, \in \mathbb{U}_{\square, \mathrm{c}} \times \mathbb{U}_{\square, \mathrm{s}, \mathrm{l}} \times \mathbb{U}_{\square, \mathrm{s}, \perp}$ satisfy the canonical equilibrium equations

$$
\begin{gathered}
\int_{\Omega_{\square, \mathrm{c}}} t_{\mathrm{c}} \boldsymbol{\sigma}_{\mathrm{c}}:\left[\delta \boldsymbol{u}_{\mathrm{c}} \otimes \nabla\right] \mathrm{d} \Omega \\
-\int_{\Gamma_{\square, \mathrm{int}}} b_{\mathrm{l}} \boldsymbol{e}_{\mathrm{l}} \cdot \delta \boldsymbol{u}_{\mathrm{c}}+b_{\perp} \boldsymbol{e}_{\perp} \cdot \delta \boldsymbol{u}_{\mathrm{c}} \mathrm{d} \Gamma \\
-\int_{\Gamma_{\square}} t_{\mathrm{c}} \hat{\boldsymbol{t}} \cdot \delta \boldsymbol{u}_{\mathrm{c}} \mathrm{d} \Gamma-\int_{\Omega_{\square, \mathrm{c}}} t_{\mathrm{c}} \hat{\boldsymbol{b}} \cdot \delta \boldsymbol{u}_{\mathrm{c}} \mathrm{d} \Omega=0 \quad \forall \delta \boldsymbol{u}_{\mathrm{c}} \in \mathbb{U}_{\square, \mathrm{c}}, \\
\int_{\Gamma_{\square, \mathrm{int}}} N_{\mathrm{s}} \frac{\partial \delta u_{\mathrm{s}, \mathrm{l}}}{\partial l}+\left[b_{\mathrm{l}}-\hat{b}_{\mathrm{l}}\right] \delta u_{\mathrm{s}, 1} \mathrm{~d} \Gamma-\left.\sum_{\mathrm{i}}\left(\hat{R}_{\mathrm{L}} \delta u_{\mathrm{s}, 1}\right)\right|_{x_{\mathrm{i}}}=0 \quad \forall \delta u_{\mathrm{s}, 1} \in \mathbb{U}_{\square, \mathrm{s}, 1}, \\
\int_{\Gamma_{\square, \mathrm{int}}}-M_{\mathrm{s}} \frac{\partial^{2} \delta u_{\mathrm{s}, \perp}}{\partial l^{2}}+b_{\perp} \delta u_{\mathrm{s}, \perp} \mathrm{d} \Gamma-\left.\sum_{\mathrm{i}}\left(\hat{R}_{\perp} \delta u_{\mathrm{s}, \perp}+\hat{R}_{\mathrm{M}} \frac{\partial \delta u_{\mathrm{s}, \perp}}{\partial l}\right)\right|_{x_{\mathrm{i}}}=0 \quad \forall \delta u_{\mathrm{s}, \perp} \in \mathbb{U}_{\square, \mathrm{s}, \perp},
\end{gathered}
$$

for suitable trial and test sets $\mathbb{U}_{\square, \mathrm{c}}, \mathbb{U}_{\square, \mathrm{s}, \mathrm{l}}, \mathbb{U}_{\mathrm{s}, \perp}$ :

$$
\begin{aligned}
\mathbb{U}_{\square, \mathrm{c}} & =\left\{\boldsymbol{u}(\boldsymbol{x}): \Omega_{\square} \mapsto \mathbb{R}^{2}, \int_{\Omega_{\square}} \boldsymbol{u}^{2}+[\boldsymbol{u} \otimes \boldsymbol{\nabla}]^{2} \mathrm{~d} \Omega<\infty\right\}, \\
\mathbb{U}_{\square, \mathrm{s}, \mathrm{l}} & =\left\{v(l): \Gamma_{\square, \text { int }} \mapsto \mathbb{R}, \int_{\Gamma_{\square, \mathrm{int}}} v^{2}+\left(\frac{\partial v}{\partial l}\right)^{2} \mathrm{~d} \Gamma<\infty\right\}, \\
\mathbb{U}_{\square, \mathrm{s}, \perp} & =\left\{v(l): \Gamma_{\square, \text { int }} \mapsto \mathbb{R}, \int_{\Gamma_{\square, \mathrm{int}}} v^{2}+\left(\frac{\partial v}{\partial l}\right)^{2}+\left(\frac{\partial^{2} v}{\partial l^{2}}\right)^{2} \mathrm{~d} \Gamma<\infty\right\} .
\end{aligned}
$$

Here, the loads (per unit length) $b_{1}$ and $b_{\perp}$ are the interaction forces between concrete and reinforcement. In addition, there is a body force (per unit volume) $\hat{\boldsymbol{b}}$ acting on the concrete, and a line load (per unit length) $\hat{b}_{1}$ acting on the reinforcement. At the boundary $\Gamma_{\square}$, the traction $\hat{\boldsymbol{t}}$ is acting on the concrete. At the endpoints of each reinforcement bar, denoted by the set $\left\{\boldsymbol{x}_{i}\right\}, \hat{R}_{\mathrm{L}}, \hat{R}_{\perp}$, and $\hat{R}_{\mathrm{M}}$ are the discrete end forces, cf. Figure 4 .

\subsubsection{RVE problem formulation}

To complete the RVE problem, the constraints are imposed via Lagrange multipliers. In this case the effective slip and slip gradient are imposed on the RVE in a weak (average) sense, i.e., by a Neumann boundary condition. In short, we require that the effective (homogenised) reinforcement slip and its gradient are equal to the imposed large-scale variables $\overline{\boldsymbol{s}}$ and $\overline{\boldsymbol{g}}$, namely

$$
\begin{array}{r}
-\left[\overline{\boldsymbol{u}}_{\square, \mathrm{s}}\left(\boldsymbol{u}_{\mathrm{s}}\right)-\overline{\boldsymbol{u}}_{\square, \mathrm{c}}\left(\boldsymbol{u}_{\mathrm{c}}\right)\right] \cdot \delta \hat{\boldsymbol{\tau}}=-\overline{\boldsymbol{s}} \cdot \delta \hat{\boldsymbol{\tau}}, \\
-\left[\overline{\boldsymbol{g}}_{\mathrm{d}, \square, \mathrm{s}}\left(\boldsymbol{u}_{\mathrm{s}}\right)-\overline{\boldsymbol{g}}_{\mathrm{d}, \square, \mathrm{c}}\left(\boldsymbol{u}_{\mathrm{c}}\right)\right]: \delta \hat{\boldsymbol{\sigma}}_{\mathrm{s}}=-\overline{\boldsymbol{g}}_{\mathrm{d}}: \delta \hat{\boldsymbol{\sigma}}_{\mathrm{s}} .
\end{array}
$$


For the displacement field in concrete, we employ classical Dirichlet boundary conditions, i.e., the fluctuation field vanishes at the boundary of the RVE and deformation there is steered by the effective strain $\bar{\varepsilon}$. The RVE problem can then be expressed as: Find $\boldsymbol{u}_{\mathrm{c}}, u_{\mathrm{s}, 1}$, $u_{\mathrm{s}, \perp}, \lambda, \hat{\boldsymbol{\tau}}, \hat{\boldsymbol{\sigma}}_{\mathrm{s}} \in \mathbb{U}_{\square, \mathrm{c}}^{D}(\overline{\boldsymbol{\varepsilon}}) \times \mathbb{U}_{\square, \mathrm{s}, \mathrm{l}} \times \mathbb{U}_{\square, \mathrm{s}, \perp} \times \mathbb{L}_{\square} \times \mathbb{R}^{2} \times \mathbb{R}_{\mathrm{d}}^{2 \times 22}$ such that

$$
\begin{aligned}
& \int_{\Omega_{\square, \mathrm{c}}} t_{\mathrm{c}} \boldsymbol{\sigma}_{\mathrm{c}}:\left[\delta \boldsymbol{u}_{\mathrm{c}} \otimes \nabla\right] \mathrm{d} \Omega-\int_{\Gamma_{\square, \mathrm{int}}} S_{\mathrm{s}} t_{\Gamma} \boldsymbol{e}_{\mathrm{l}} \cdot \delta \boldsymbol{u}_{\mathrm{c}} \mathrm{d} \Gamma-\int_{\Gamma_{\square, \mathrm{int}}} \lambda \boldsymbol{e}_{\perp} \cdot \delta \boldsymbol{u}_{\mathrm{c}} \mathrm{d} \Gamma \\
& +\int_{\Omega_{\square, \mathrm{c}}} \delta \boldsymbol{u}_{\mathrm{c}} \mathrm{d} \Omega \cdot \hat{\boldsymbol{\tau}}=0 \quad \forall \delta \boldsymbol{u}_{\mathrm{c}} \in \mathbb{U}_{\square, \mathrm{c}}^{D}(\mathbf{0}), \\
& \int_{\Gamma_{\square, \mathrm{int}}} N_{\mathrm{s}} \frac{\partial \delta u_{\mathrm{s}, 1}}{\partial l} \mathrm{~d} \Gamma+\int_{\Gamma_{\square, \mathrm{int}}} S_{\mathrm{s}} t_{\Gamma} \delta u_{\mathrm{s}, 1} \mathrm{~d} \Gamma-\int_{\Gamma_{\square, \mathrm{int}}} S_{\mathrm{s}} \delta u_{\mathrm{s}, l} \boldsymbol{e}_{1} \mathrm{~d} \Gamma \cdot \boldsymbol{C}_{\mathrm{s}} \cdot \hat{\boldsymbol{\tau}} \\
& -\int_{\Gamma_{\square, \mathrm{int}}} S_{\mathrm{s}} \frac{\partial \delta u_{\mathrm{s}, 1}}{\partial l} \boldsymbol{e}_{\mathrm{l}} \otimes \boldsymbol{e}_{\mathrm{l}} \mathrm{d} \Gamma \cdot \boldsymbol{C}_{\mathrm{s}}: \hat{\boldsymbol{\sigma}}_{\mathrm{s}}=0 \quad \forall \delta u_{\mathrm{s}, l} \in \mathbb{U}_{\square, \mathrm{s}, l} \\
& -\int_{\Gamma_{\square, \mathrm{int}}} M_{\mathrm{s}} \frac{\partial^{2} \delta u_{\mathrm{s}, \perp}}{\partial l^{2}} \mathrm{~d} \Gamma+\int_{\Gamma_{\square, \mathrm{int}}} \lambda \delta u_{\mathrm{s}, \perp} \mathrm{d} \Gamma=0 \quad \forall \delta u_{\mathrm{s}, \perp} \in \mathbb{U}_{\square, \mathrm{s}, \perp}, \\
& \int_{\Gamma_{\square, \mathrm{int}}}\left[u_{\mathrm{s}, \perp}-\boldsymbol{e}_{\perp} \cdot \boldsymbol{u}_{\mathrm{c}}\right] \delta \lambda \mathrm{d} \Gamma=0 \quad \forall \delta \lambda \in \mathbb{L}_{\square} \\
& -\left[\int_{\Gamma_{\square, \mathrm{int}}} S_{\mathrm{s}} u_{\mathrm{s}, l} \boldsymbol{e}_{\mathrm{l}} \mathrm{d} \Gamma \cdot \boldsymbol{C}_{\mathrm{s}}-\int_{\Omega_{\square}} \boldsymbol{u}_{\mathrm{c}} \mathrm{d} \Omega\right] \cdot \delta \hat{\boldsymbol{\tau}}=-\overline{\boldsymbol{s}} \cdot \delta \hat{\boldsymbol{\tau}}\left|\Omega_{\square}\right| \quad \forall \delta \hat{\boldsymbol{\tau}} \in \mathbb{R}^{2}, \\
& -\left[\int_{\Gamma_{\square, \mathrm{int}}} S_{\mathrm{s}} \frac{\partial u_{\mathrm{s}, 1}}{\partial l} \boldsymbol{e}_{\mathrm{l}} \otimes \boldsymbol{e}_{\mathrm{l}} \mathrm{d} \Gamma \cdot \boldsymbol{C}_{\mathrm{s}}\right]: \delta \hat{\boldsymbol{\sigma}}_{\mathrm{s}}=-\left[\overline{\boldsymbol{g}}_{\mathrm{d}}+\overline{\boldsymbol{\varepsilon}}\right]: \delta \hat{\boldsymbol{\sigma}}_{\mathrm{s}}\left|\Omega_{\square}\right| \quad \forall \delta \hat{\boldsymbol{\sigma}}_{\mathrm{s}} \in \mathbb{R}_{\mathrm{d}}^{2 \times 2},
\end{aligned}
$$

for the suitable trial set

$$
\mathbb{U}_{\square, \mathrm{c}}^{D}(\overline{\boldsymbol{\varepsilon}})=\left\{\boldsymbol{u} \in \mathbb{U}_{\square, \mathrm{c}}: \boldsymbol{u}=\overline{\boldsymbol{\varepsilon}} \cdot[\boldsymbol{x}-\overline{\boldsymbol{x}}] \text { on } \Gamma_{\square}\right\},
$$

and the pertinent test spaces. This formulation does indeed satisfy $\mathcal{A}^{*}\left(\boldsymbol{u}_{\mathrm{c}}^{s}, \boldsymbol{u}_{\mathrm{s}}^{s}\right)=\mathbf{0}$, thus guaranteeing the well-posedness of the scale transition discussed in Section 3.2.

Remark:

In deriving Equations (63) and (68), it was explicilty used that

$$
\frac{1}{\left|\Omega_{\square}\right|} \int_{\Omega_{\square, \mathrm{c}}} \boldsymbol{u}_{\mathrm{c}} \otimes \nabla \mathrm{d} \Omega=\overline{\boldsymbol{\varepsilon}} \quad \text { and } \quad \frac{1}{\left|\Omega_{\square}\right|} \int_{\Omega_{\square, \mathrm{c}}} \delta \boldsymbol{u}_{\mathrm{c}} \otimes \nabla \mathrm{d} \Omega=\mathbf{0},
$$

for $\boldsymbol{u}_{\mathrm{c}} \in \mathbb{U}_{\square, \mathrm{c}}^{D}(\overline{\boldsymbol{\varepsilon}})$ and $\delta \boldsymbol{u}_{\mathrm{c}} \in \mathbb{U}_{\square, \mathrm{c}}^{D}(\mathbf{0})$, respectively.

\footnotetext{
${ }^{2} \mathbb{R}_{\mathrm{d}}^{2 \times 2}$ is a diagonal second order tensor with respect to orthogonal reinforcement directions $\left(\boldsymbol{e}_{1,1}, \boldsymbol{e}_{1,2}\right)$
} 


\subsubsection{Physical interpretation of Lagrange multipliers}

By comparing the RVE problem formulated in Section 3.4.2 with its canonical form stated in Section 3.4.1, we can identify physical interpretation of the introduced Lagrange multipliers in the proposed formulation. First, we note that the interaction forces between concrete and reinforcement are exactly those presented in the single scale (reference) problem in Eqs. (7)-(10),

$$
\begin{aligned}
b_{1} & =S_{\mathrm{s}} t_{\Gamma}, \\
b_{\perp} & =\lambda,
\end{aligned}
$$

where we recall that $\lambda$ is a Lagrange multiplier preventing discontinuity in the transverse direction. Secondly, we identify the introduced body forces as

$$
\begin{aligned}
& \hat{\boldsymbol{b}}=-\frac{\hat{\boldsymbol{\tau}}}{t_{\mathrm{c}}}, \\
& \hat{b}_{\mathrm{l}}=S_{\mathrm{s}} \boldsymbol{e}_{\mathrm{l}} \cdot \boldsymbol{C}_{\mathrm{s}} \cdot \hat{\boldsymbol{\tau}},
\end{aligned}
$$

i.e., the condition on the effective slip is met by introducing "fictitious" body forces on the concrete and reinforcement bars, respectively. These forces are in equilibrium. Finally, we identify the end forces on the reinforcement bars as

$$
\begin{aligned}
\hat{R}_{\mathrm{L}} & =\left[S_{\mathrm{s}} e_{\mathrm{nl}} \boldsymbol{e}_{\mathrm{l}} \cdot \boldsymbol{C}_{\mathrm{s}} \otimes \boldsymbol{e}_{\mathrm{l}}\right]: \hat{\boldsymbol{\sigma}}_{\mathrm{s}} \\
\hat{R}_{\perp}= & \hat{R}_{\mathrm{M}}=0
\end{aligned}
$$

where $e_{\mathrm{nl}}= \pm 1$ at start- and endpoints as introduced in conjunction to Equation (47). Hence, axial forces pertinent to the constraint on the gradient of slip are applied at the endpoints, whereas no transverse forces or bending moments act on the reinforcement bar ends. The boundary traction acting on the concrete, $\hat{\boldsymbol{t}}$, will be the (fluctuating) reaction caused by the Dirichlet condition on $\boldsymbol{u}_{\mathrm{c}}$ on $\Gamma_{\square}$.

\subsubsection{Effective work conjugates}

Further analysis of Equation (64) together with the constraint equations (61)-(62) reveals the quantitative link between the Lagrange multipliers $\hat{\boldsymbol{\tau}}, \hat{\boldsymbol{\sigma}}_{\mathrm{s}}$ and the effective transfer and reinforcement stresses $\overline{\boldsymbol{\tau}}_{\mathrm{b}}$ and $\overline{\boldsymbol{\sigma}}_{\mathrm{s}}$. First, by considering Eq. (64) for the test function $\delta u_{\mathrm{s}, \mathrm{l}}=\boldsymbol{e}_{\mathrm{l}} \cdot \delta \overline{\boldsymbol{u}}$, for a constant $\delta \overline{\boldsymbol{u}}$, we obtain the relation

$$
\delta \overline{\boldsymbol{u}} \cdot \int_{\Gamma_{\square, \mathrm{int}}} S_{\mathrm{s}} t_{\Gamma} \boldsymbol{e}_{\mathrm{l}} \mathrm{d} \Gamma=\delta \overline{\boldsymbol{u}} \cdot \int_{\Gamma_{\square, \mathrm{int}}} S_{\mathrm{s}} \boldsymbol{e}_{\mathrm{l}} \otimes \boldsymbol{e}_{\mathrm{l}} \mathrm{d} \Gamma \cdot \boldsymbol{C}_{\mathrm{s}} \cdot \hat{\boldsymbol{\tau}} \quad \forall \delta \overline{\boldsymbol{u}} \in \mathbb{R}^{2}
$$

By identifying the effective transfer stress from Eq. (51), and by using the identity

$$
\frac{1}{\left|\Omega_{\square}\right|} \int_{\Gamma_{\square, \mathrm{int}}} S_{\mathrm{s}} \boldsymbol{e}_{\mathrm{l}} \otimes \boldsymbol{e}_{\mathrm{l}} \mathrm{d} \Gamma \cdot \boldsymbol{C}_{\mathrm{s}}=\mathbb{I}
$$


cf. Appendix A, we obtain the result

$$
\bar{\tau}_{\mathrm{b}}=\hat{\tau}
$$

Secondly, selecting the test function $\delta u_{\mathrm{s}, \mathrm{l}}=\boldsymbol{e}_{\mathrm{l}} \cdot \delta \overline{\boldsymbol{g}}_{\mathrm{d}} \cdot[\boldsymbol{x}-\overline{\boldsymbol{x}}]$ in Equation (64), for a constant $\delta \overline{\boldsymbol{g}}_{\mathrm{d}} \in \mathbb{R}_{\mathrm{d}}^{2 \times 2}$, we obtain the relation

$$
\begin{gathered}
\delta \overline{\boldsymbol{g}}_{\mathrm{d}}:\left[\int_{\Gamma_{\square, \mathrm{int}}} N_{\mathrm{s}} \boldsymbol{e}_{\mathrm{l}} \otimes \boldsymbol{e}_{\mathrm{l}} \mathrm{d} \Gamma+\int_{\Gamma_{\square, \mathrm{int}}} S_{\mathrm{s}} t_{\Gamma} \boldsymbol{e}_{\mathrm{l}} \otimes[\boldsymbol{x}-\overline{\boldsymbol{x}}] \mathrm{d} \Gamma\right] \\
=\delta \overline{\boldsymbol{g}}_{\mathrm{d}}:\left[\int_{\Gamma_{\square, \mathrm{int}}} S_{\mathrm{s}} \boldsymbol{e}_{\mathrm{l}} \otimes[\boldsymbol{x}-\overline{\boldsymbol{x}}] \otimes \boldsymbol{e}_{\mathrm{l}} \mathrm{d} \Gamma \cdot \boldsymbol{C}_{\mathrm{s}} \cdot \hat{\boldsymbol{\tau}}+\int_{\Gamma_{\square, \mathrm{int}}} S_{\mathrm{s}} \boldsymbol{e}_{\mathrm{l}} \otimes \boldsymbol{e}_{\mathrm{l}} \otimes \boldsymbol{e}_{\mathrm{l}} \otimes \boldsymbol{e}_{\mathrm{l}} \mathrm{d} \Gamma \cdot \boldsymbol{C}_{\mathrm{s}}: \hat{\boldsymbol{\sigma}}_{\mathrm{s}}\right] \quad \forall \delta \overline{\boldsymbol{g}}_{\mathrm{d}} \in \mathbb{R}_{\mathrm{d}}^{2 \times 2} .
\end{gathered}
$$

As shown in Appendix A, the integrals

$$
\int_{\Gamma_{\square, \mathrm{int}}} S_{\mathrm{s}} \boldsymbol{e}_{\mathrm{l}} \otimes[\boldsymbol{x}-\overline{\boldsymbol{x}}] \otimes \boldsymbol{e}_{\mathrm{l}} \mathrm{d} \Gamma=0 \quad \text { and } \quad \frac{1}{\left|\Omega_{\square}\right|} \int_{\Gamma_{\square, \mathrm{int}}} S_{\mathrm{s}} \boldsymbol{e}_{\mathrm{l}} \otimes \boldsymbol{e}_{\mathrm{l}} \otimes \boldsymbol{e}_{\mathrm{l}} \otimes \boldsymbol{e}_{\mathrm{l}} \mathrm{d} \Gamma \cdot \boldsymbol{C}_{\mathrm{s}}=\mathbb{I}_{\mathrm{d}},
$$

where we recall that $\boldsymbol{C}_{\mathrm{s}}=\boldsymbol{C}_{\mathrm{g}}$. Using these identities in Equation (77), together with the definition of the effective reinforcement stress from Equation (52), we obtain the result

$$
\overline{\boldsymbol{\sigma}}_{\mathrm{s}}=\hat{\boldsymbol{\sigma}}_{\mathrm{s}}
$$

In summary, we conclude that the Lagrange multipliers pertaining to the constraints on effective slip and slip gradient, presented in Eqs. (61) and (62), are indeed identical to the work conjugates being the transfer and reinforcement stress, respectively. It is noteworthy that the results from Sections (3.4.1)-(3.4.4) correspond to the Hill-Mandel principle of energy equivalence, see e.g. [34],

$$
\begin{aligned}
& \frac{1}{\left|\Omega_{\square}\right|}\left[\int_{\Omega_{\square, \mathrm{c}}} t_{\mathrm{c}} \boldsymbol{\sigma}_{\mathrm{c}}:\left[\delta \boldsymbol{u}_{\mathrm{c}} \otimes \boldsymbol{\nabla}\right] \mathrm{d} \Omega+\int_{\Gamma_{\square, \mathrm{int}}} S_{\mathrm{s}} t_{\Gamma}\left[\delta u_{\mathrm{s}, l}-\boldsymbol{e}_{\mathrm{l}} \cdot \delta \boldsymbol{u}_{\mathrm{c}} \mathrm{d} \Gamma\right]\right. \\
& \left.\quad+\int_{\Gamma_{\square, \text { int }}} N_{\mathrm{s}} \frac{\partial \delta u_{\mathrm{s}, 1}}{\partial l}-M_{\mathrm{s}} \frac{\partial^{2} \delta u_{\mathrm{s}, \perp}}{\partial l^{2}} \mathrm{~d} \Gamma\right]=\overline{\boldsymbol{\sigma}}: \delta \overline{\boldsymbol{\varepsilon}}+\overline{\boldsymbol{\sigma}}_{\mathrm{s}}: \delta \overline{\boldsymbol{g}}_{\mathrm{d}}+\overline{\boldsymbol{\tau}}_{\mathrm{b}} \cdot \delta \overline{\boldsymbol{s}}
\end{aligned}
$$

\section{Unit cell response at reinforcement pull-through}

The performance of the novel formulation of prescribing the macroscopic slip and slip gradient inside the RVE volume was studied by means of a series of simulations. The so-called pull-through tests are structural simulations performed on the RVEs, where the macroscopic slip is gradually increased, so that the reinforcement bars are eventually pulled out from the concrete. Such tests were already performed by the authors in [22], with the effective reinforcement slip being prescribed at the RVE boundary, but nothing about the subscale variation was specified. It is noteworthy, that such formulation did not require any 
Table 1: Geometry of the unit cells.

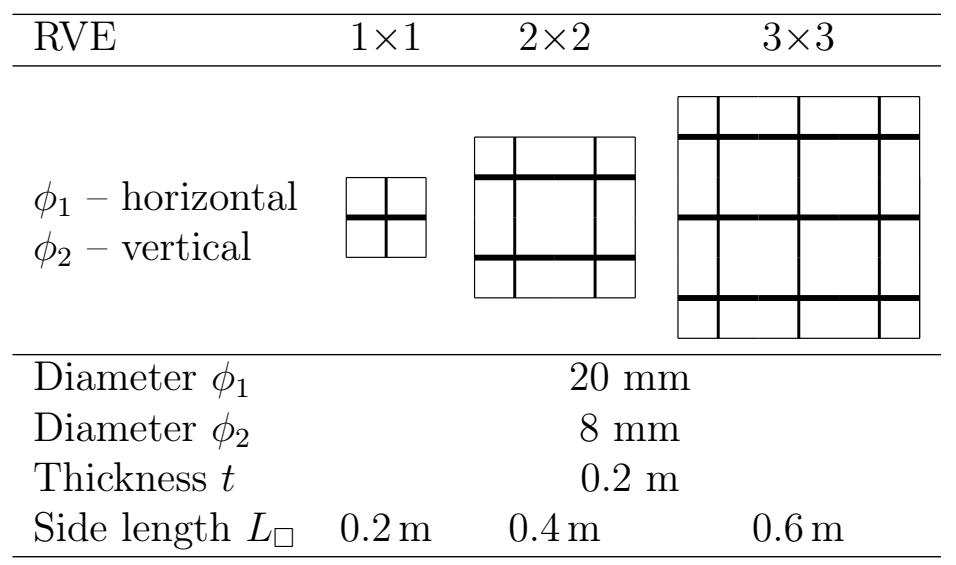

volumetric/subscale definition of the effective variable. It was found out that the effective work conjugate (transfer stress) was highly RVE size dependent, i.e., the definition of the effective variable was not objective. In this section, the pull-through tests are repeated, and the results obtained when prescribing the slip at the boundary are compared with the results obtained when prescribing the slip in volume.

\subsection{Test layout}

Three different two-dimensional unit cells were considered for the test, each one in the shape of square and with equal number of reinforcement bars in the horizontal and vertical direction, cf. Table 1. Note that the spacing of reinforcement bars is equal in both directions for all unit cells.

A graphical summary of the constitutive models used for the individual materials together with the values of material parameters, are presented in Table 2. An isotropic continuum damage model, commonly known as the Mazars' model [35, 36], was used. In tension, an exponential damage law was used [37]. In compression, the classical Mazars' damage formulation was kept. Bilinear quadrilateral elements with size of $0.02 \mathrm{~m}$ were used. The reinforcement was modelled as an elastoplastic material with strain hardening with the use of beam elements. Standard interface elements were used to model steel/concrete bond, according to the bond-slip input specified in Table 2 .

In the simulations, the macroscopic reinforcement slip was imposed on the RVEs in horizontal direction only, i.e., in steps of $\Delta \overline{\boldsymbol{s}}=\left[5 \times 10^{-5} \mathrm{~m}, 0\right]^{T}$. The effective strain, $\overline{\boldsymbol{\varepsilon}}$, and the effective slip gradient, $\overline{\boldsymbol{g}}$, were kept constant at zero in all directions. The macroscopic slip was prescribed using two alternatives: 1) only on the boundary of the RVE, and 2) in the RVE volume. The simulations were performed in the open source $\mathrm{C}++$ code $\mathrm{OOFEM}$ (www. oof em.org) [38].

\subsection{Results and discussion}

The effective transfer stress, $\overline{\boldsymbol{\tau}}_{\mathrm{b}}$, was computed after solving the subscale boundary value problem on the unit cell. It is presented as a function of the macroscopic slip in Figure 5a 
Table 2: Material parameters for the concrete, steel, and the interface.

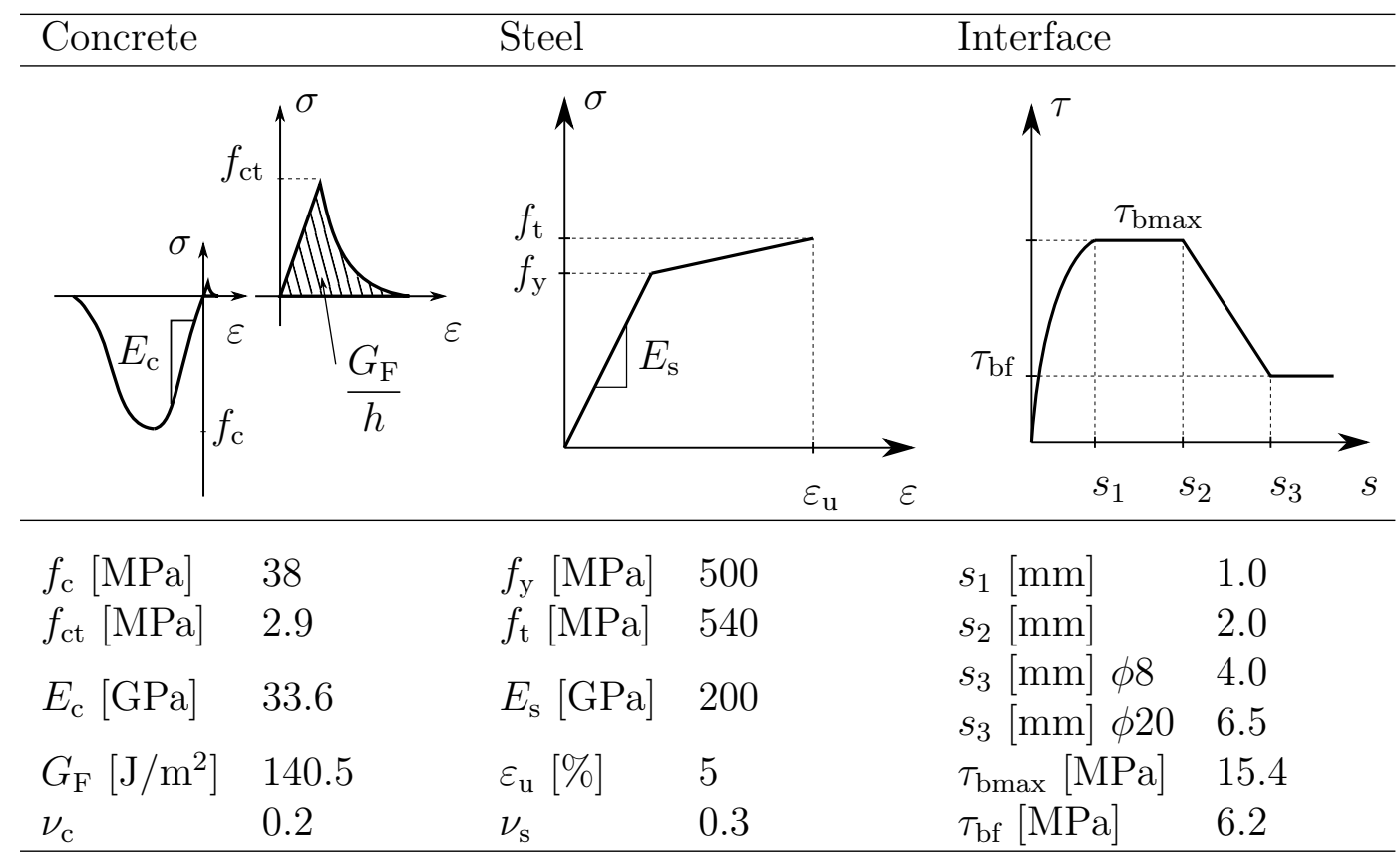

for both boundary and volumetric definition. The largest eigenvalue of the initial sensitivity tensor associated with effective transfer stress and effective slip is plotted for the varying size of the unit cell in Figure 5b. The corresponding local slip profiles along the rebars taken at the macroscopic slip of $1.5 \mathrm{~mm}$ (top plateau of bond-slip input) and $8 \mathrm{~mm}$ (bottom plateau of bond-slip input) are presented in Figure 6a and Figure 6b, respectively.

Comparing the transfer stress-macroscopic slip results from Figure 5a, it can be seen that imposing the effective slip weakly via Lagrange multipliers results in RVE size independent response; the bond-slip input is recovered directly for all unit cells. This was clearly not the case when imposing the macroscopic slip at the boundary only; similar recovery took place only for the smallest RVE. Due to introduced volumetric constraints, the slip distribution along the rebars is almost constant along the bars. Moreover, the average value of the local slip, computed according to the definition (40), is equal to the macroscopic value. For the case when slip was prescribed only at the boundary, the local slip quickly reduced inside the rebar, especially for longer bars. As a result, the average value was not equal to the imposed one (for the larger RVEs), and thus the effective work conjugate, $\overline{\boldsymbol{\tau}}_{\mathrm{b}}$ was also smaller than it would be expected, for the applied macroscopic slip. When the macroscopic slip is prescribed in volume, the largest eigenvalue of the initial sensitivity tensor associated with the transfer stress and slip reaches a converged value already for small unit cells and does not change very much with size of the RVE. When the slip is prescribed only at boundary, larger RVE (longer rebar) means lower slip (bond stress) in the interior of the rebar, thus making the transfer stress also lower. It can therefore be expected for larger RVEs to have smaller stiffness (sensitivity) in the interface, $\partial \overline{\boldsymbol{\tau}}_{\mathrm{b}} / \partial \overline{\boldsymbol{s}}$. This effect can be observed in Figure $5 \mathrm{~b}$. To summarise, the RVE size dependence (with respect to effective slip and transfer stress) was 


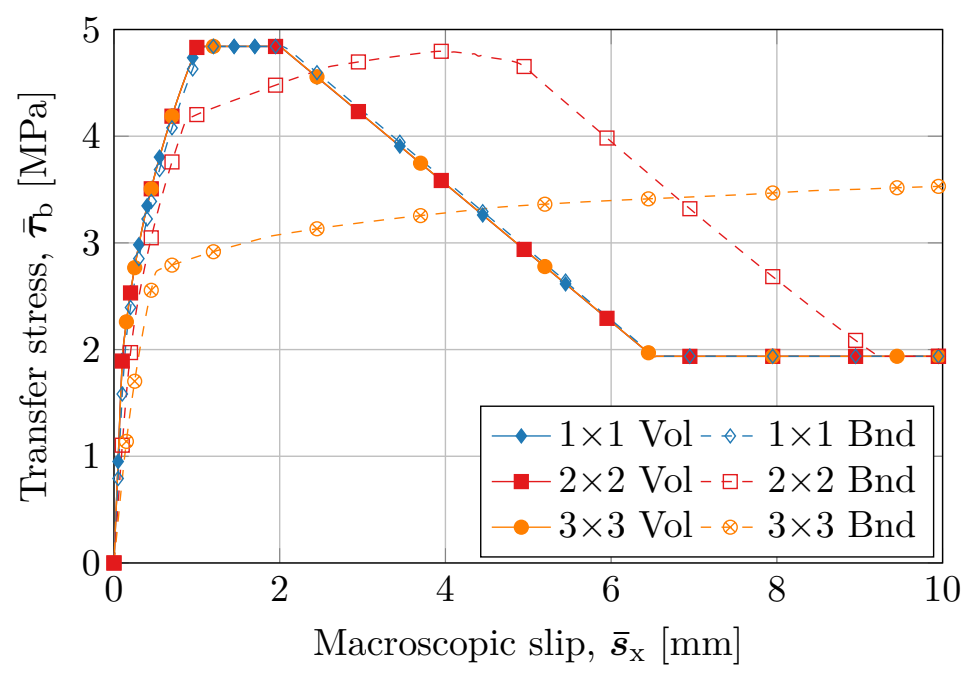

(a)

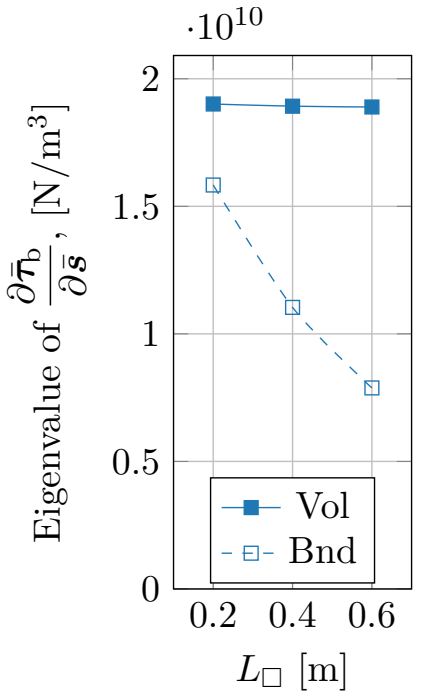

(b)

Figure 5: (a) Transfer stress-macroscopic slip relations for different unit cells obtained when prescribing the slip in RVE volume ( $\mathrm{Vol}$ ) and on RVE boundary (Bnd). (b) Variation of largest eigenvalue of the initial sensitivity tensor $\partial \bar{\tau}_{\mathrm{b}} / \partial \overline{\boldsymbol{s}}$ with the size of the unit cell.

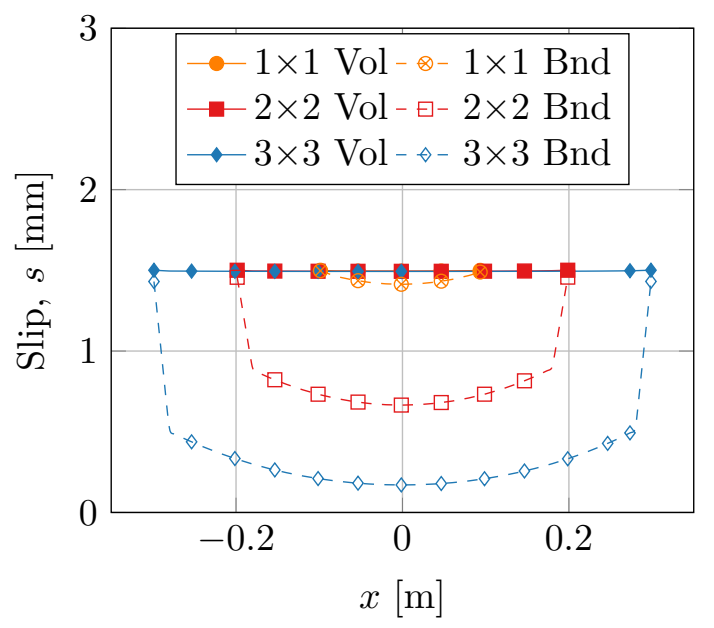

(a) $\bar{s}_{\mathrm{x}}=1.5 \mathrm{~mm}$

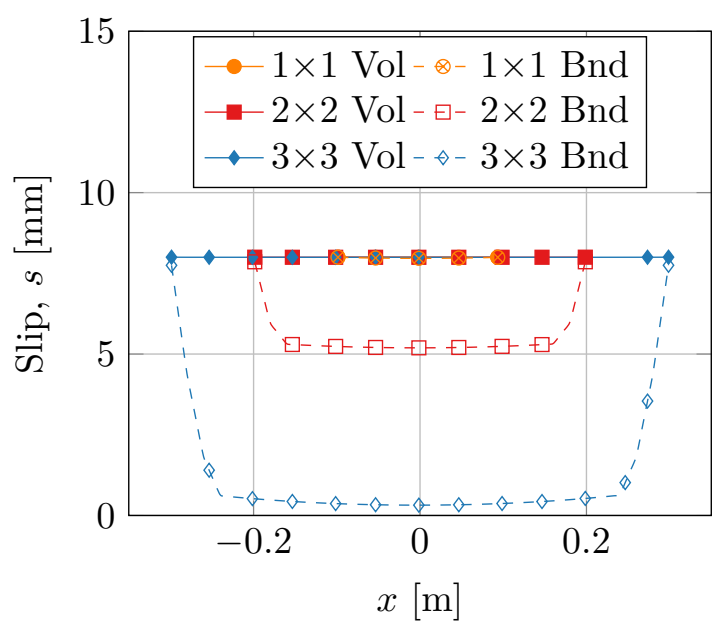

(b) $\bar{s}_{\mathrm{x}}=8 \mathrm{~mm}$

Figure 6: Reinforcement slip variation along the rebars in $1 \times 1,2 \times 2,3 \times 3$ RVEs at the macroscopic slip $1.5 \mathrm{~mm}$ (a) and $8 \mathrm{~mm}$ (b) obtained with the corresponding volumetric (Vol) and boundary (Bnd) definition of effective reinforcement slip. 


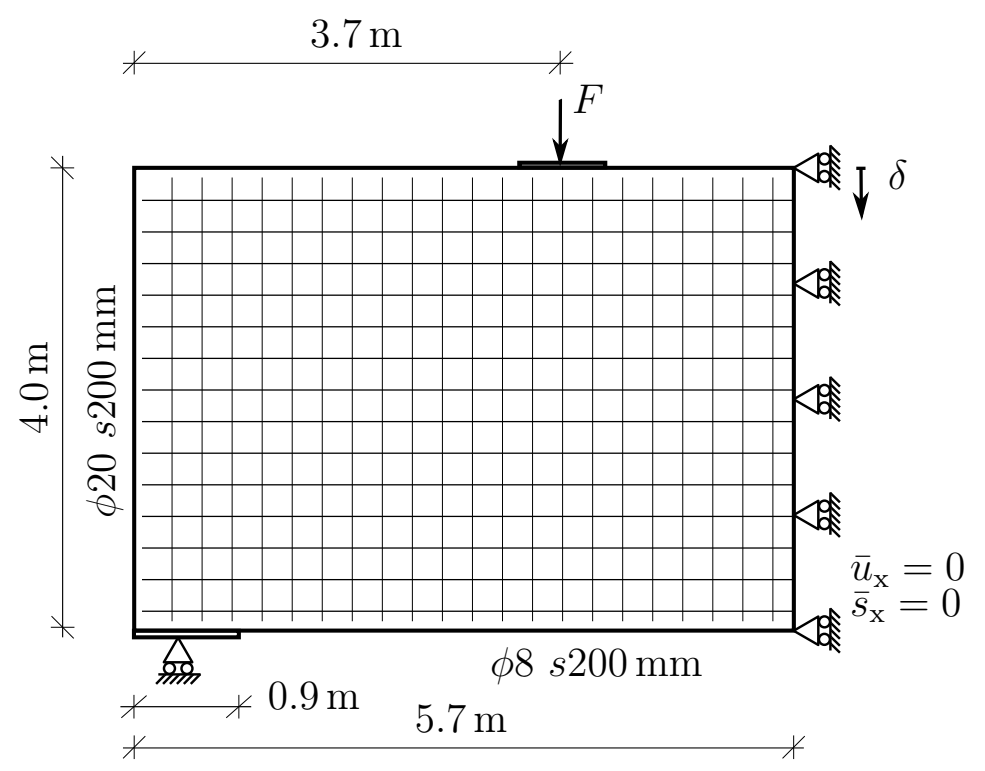

Figure 7: Schematic of the uniformly reinforced deep beam. Geometry, reinforcement layout, boundary conditions and mid-span deflection, $\delta$, are indicated.

rectified by introducing the volumetric constraints via Lagrange multipliers. In contrast to the classical boundary formulation, the interpretation of the effective reinforcement slip was no longer size dependent.

\section{Large-scale response}

A simply supported reinforced concrete deep beam under four-point bending was considered for next numerical study. The structure was uniformly reinforced in both horizontal ( $\phi 20$ bars) and vertical ( $\phi 8$ bars) directions. In view of the symmetry of the beam, it sufficed to model half of it, cf. Figure 7 for details concerning geometry and reinforcement layout. The thickness of the structure was $0.2 \mathrm{~m}$, and thus plane stress assumption was employed.

The purpose of this numerical study was to investigate the effect of prescribing the macroscopic slip in volume /rather than on boundary) on the structural response in a twoscale computational scheme. In particular, the effective strain and slip fields are of interest. The influence of the mentioned definition of effective slip on predicting the maximum crack width was also studied. The two-scale computational method used in this work was the $\mathrm{FE}^{2}$ method.

In the analysis, displacement control was used, with the vertical displacement under the loading platen was increased in steps of $0.1 \mathrm{~mm}$. In the large-scale model, 8-node biquadratic elements with 9 Gauss points were chosen. At the symmetry line, degrees of freedom corresponding to horizontal displacement and reinforcement slip were locked. Since the arrangement of reinforcement in the deep beam corresponded to the one used in RVEs described in Section 4, they were directly used to represent the substructure of the material. 
Table 3: Macroscopic mesh diameter to subscale unit cell size ratios, $h / L_{\square}$, for the $\mathrm{FE}^{2}$ analyses

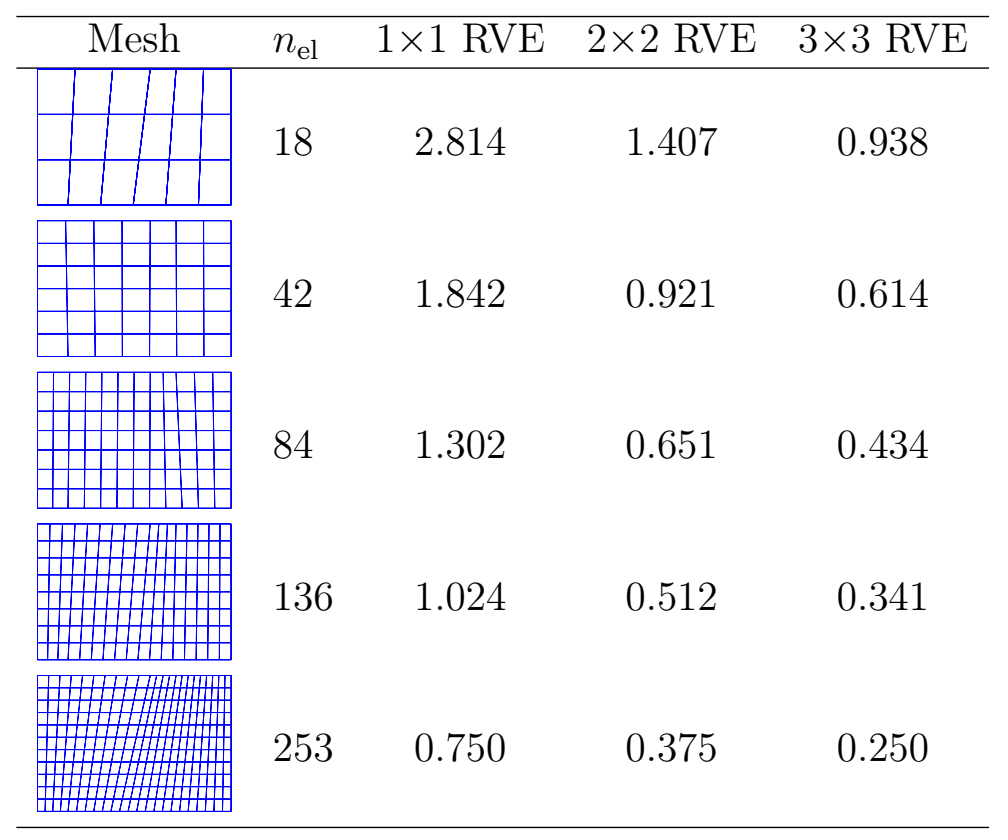

It is noteworthy, that not only the size of the RVE, but also the size of the macroscopic mesh has influence on the results, as shown by the authors in [22]. To this end, five different large-scale meshes were constructed. Sampling ratios, i.e., the ratios of the macroscopic mesh diameter (corresponding to single Gauss point) to unit cell size are given for all meshes in Table 3. For the simulations, the open source C ++ code OOFEM (www .oofem.org) [38] was used.

\subsection{Global load-deflection response}

The load-mid-span deflection relation is a global result that can be directly used to evaluate structural performance of the studied beam. These relations are presented in Figures (8)-(10) for different sizes of the unit cell and the type of definition of the macroscopic slip. The mid-span deflection was measured at the top of the beam, according to Figure 7, while the external force was computed as the reaction force at the loading platen. For convenience, a result for a single-scale analysis in full resolution is also presented, cf. [22] for more information about the single-scale model. From the graphs, a few things can be concluded. First, it is evident that the type of definition did not influence the effective loaddeflection response. Secondly, the large-scale mesh dependence of the global response was rather low. Moreover, since the macroscopic strain was prescribed on the RVEs via classical Dirichlet boundary condition, the structural stiffness (and load-deflection response) was overestimated, which was already observed by the authors in [21, 22]. Since bond-slip mechanism is very important only locally (e.g. for cracking), it could be expected that its influence on the global structural response will be marginal. 


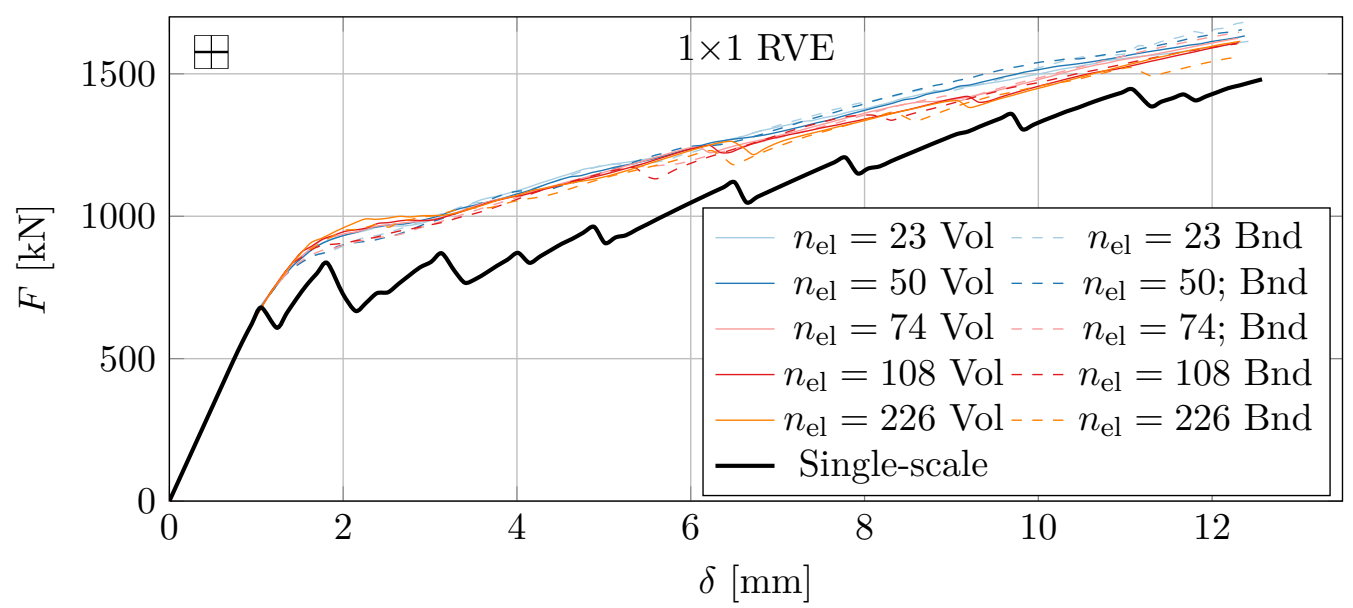

Figure 8: External load - mid-span deflection relation for two-scale analyses using $1 \times 1$ RVE and prescribing the macroscopic slip in volume ( $\mathrm{Vol}$ ) and only at boundary (Bnd) of the RVE.

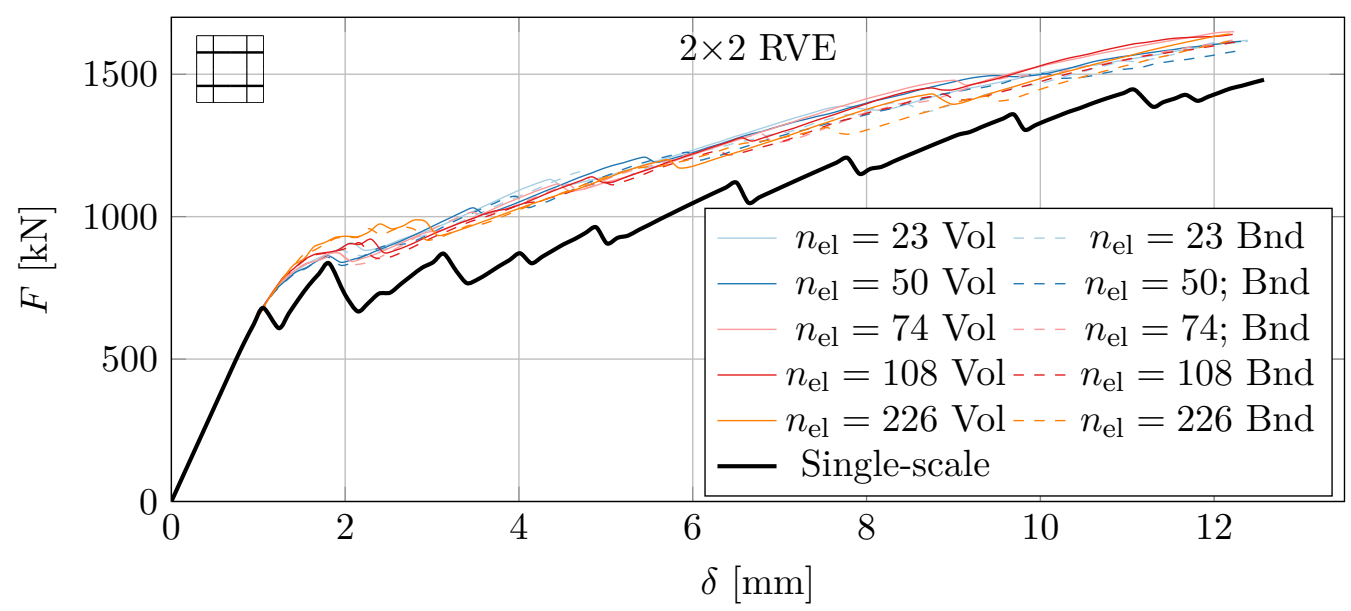

Figure 9: External load - mid-span deflection relation for two-scale analyses using $2 \times 2$ RVE and prescribing the macroscopic slip in volume ( $\mathrm{Vol}$ ) and only at boundary (Bnd) of the RVE. 


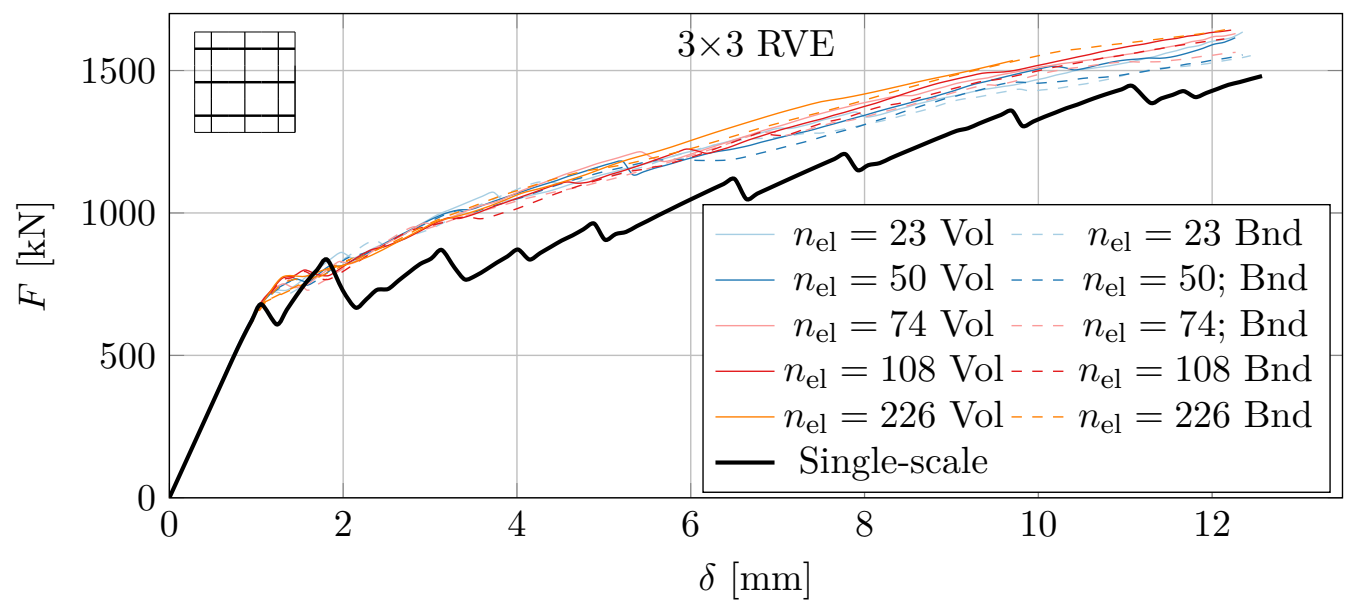

Figure 10: External load - mid-span deflection relation for two-scale analyses using $3 \times 3$ RVE and prescribing the macroscopic slip in volume ( Vol) and only at boundary (Bnd) of the RVE.

\subsection{Effective strain and slip fields}

The most interesting large-scale results are arguably the effective strain and slip fields. Figures 11a and 12a show the first principal effective strain, $\bar{\varepsilon}_{1}$, at a load step resembling serviceability limit state, i.e., after cracking of concrete, but before yielding of reinforcement. In the figures, the areas corresponding to individual Gauss points have been assigned constant strain. As already shown in [22], the incorporation of slip as another macroscopic variable results in effective strain localisation, and this effect can be observed here for both types of macroscopic slip definition. Moreover, not only the size of the unit cell, but also the resolution of macroscopic mesh was important in interpretation of the two-scale results. By studying the results, it can be inferred that prescribing the effective slip in volume of the RVE did not have a large influence on the global result. For the considered meshes, the influence of the size of the unit cell was already small for both types of boundary conditions. Overall, there was a small tendency for the effective strain obtained when prescribing the slip in volume to go from more "smeared" strain patterns to more "localised" as the size of the unit cell grows, with the process zone being more or less the same.

Locally, there is a correlation between reinforcement slip and crack patterns, with large gradient in slip signifying a crack. If the crack discontinuity is chosen to be represented weakly in model, i.e., by band of higher strains (as is the case for most smeared crack models), the slip and crack patterns should be closely related. This strain-slip interplay could also be observed for their effective counterparts in Figures $11 \mathrm{~b}$ and $12 \mathrm{~b}$, where the horizontal component of the effective slip vector was plotted for the two-scale analyses. The effective slip values were readily available at nodes after solving the large-scale problem, and could be plotted directly over the macroscopic mesh.

Even though there is a clear localisation of the effective strain reflected by the macroscopic slip field, it comes from the governing partial differential equations allowing for slip transfer across large-scale elements. As such, it it not physically correct to interpret the 


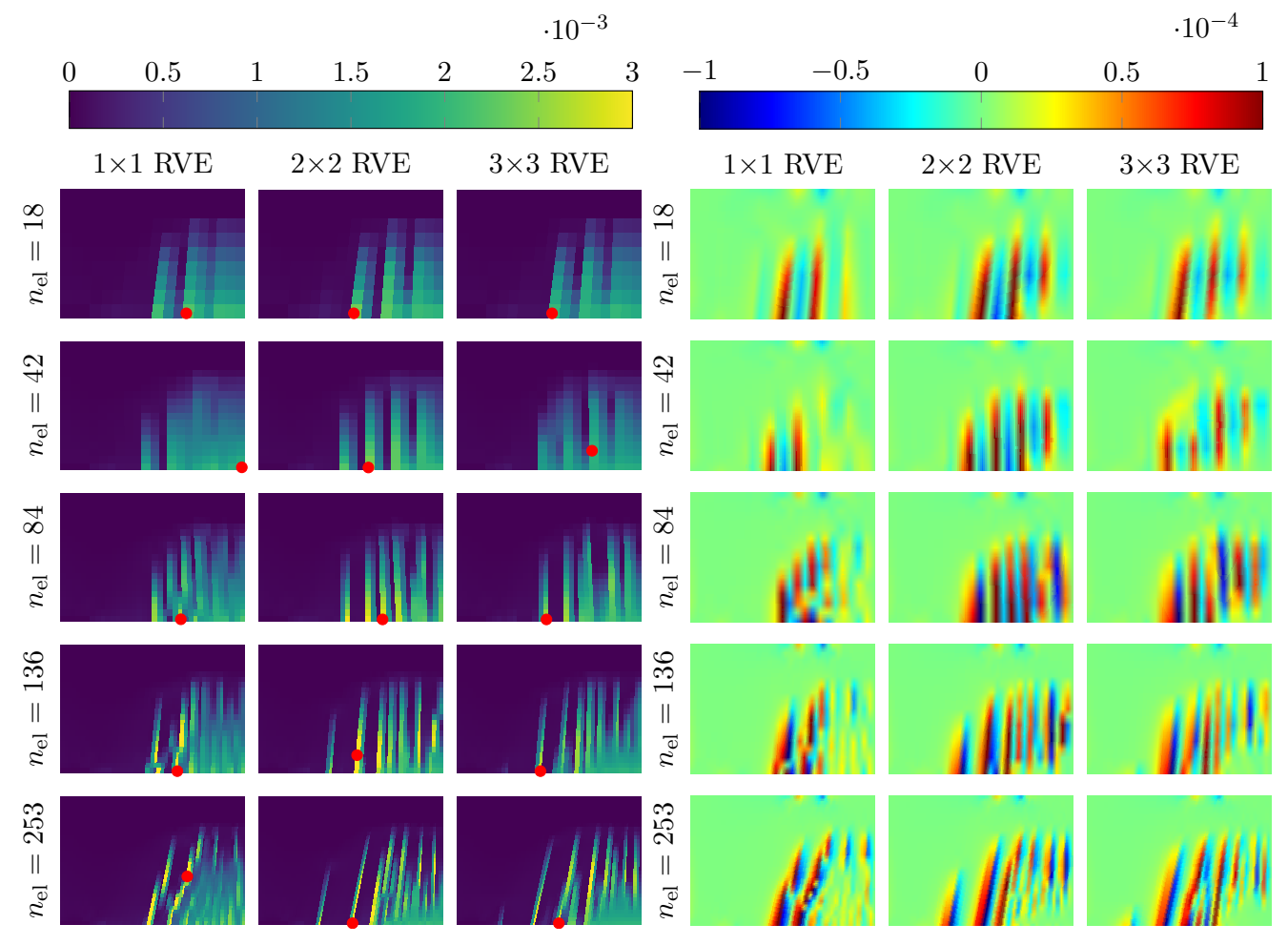

(a) $\bar{\varepsilon}_{1}$

(b) $\bar{s}_{\mathrm{X}}$

Figure 11: First principal strain (a) and horizontal slip (b), in the large-scale domain obtained when prescribing the effective slip in volume of the RVE. Location of the largest principal strain indicated with dot. 


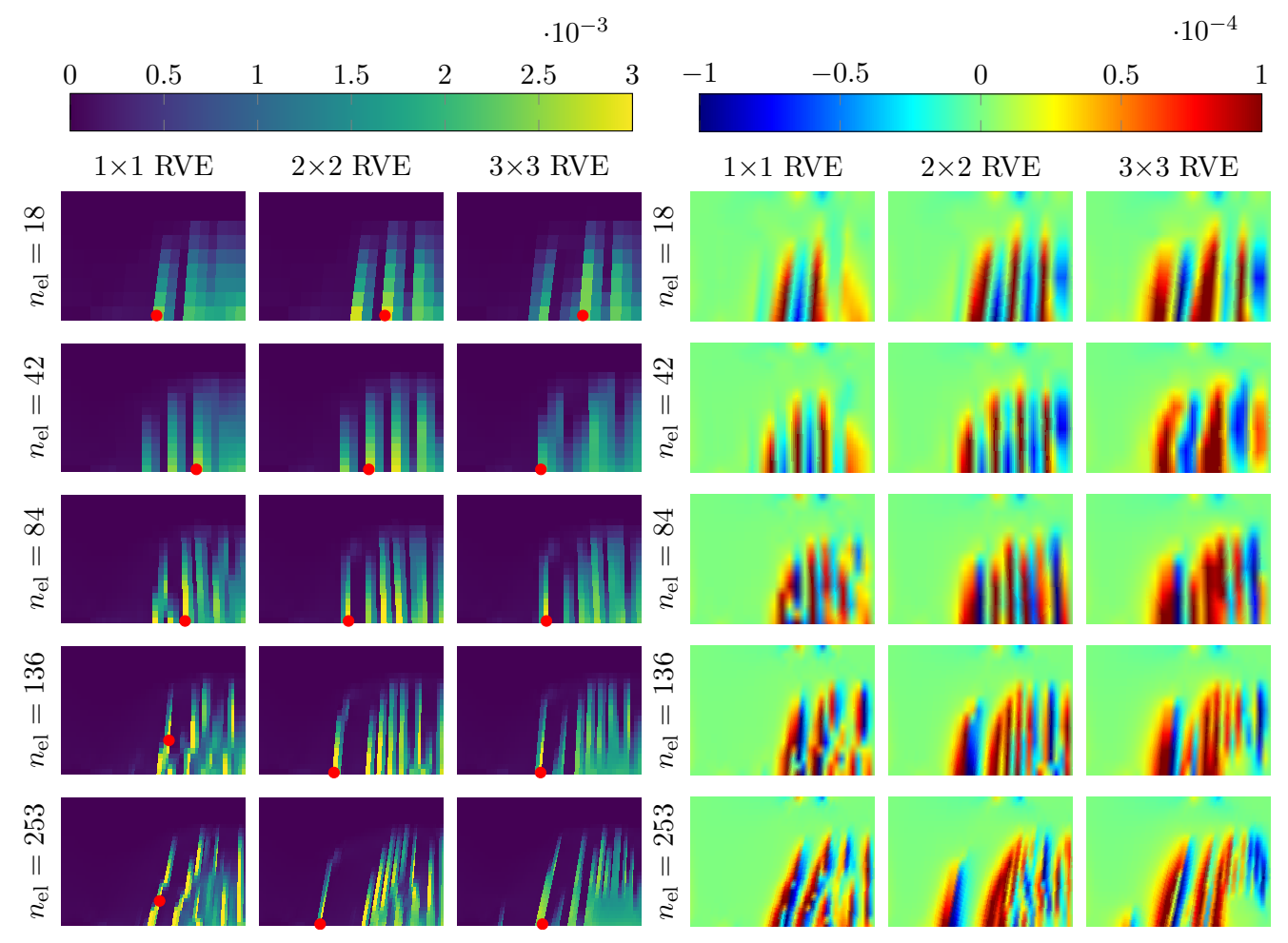
(a) $\bar{\varepsilon}_{1}$
(b) $\bar{s}_{\mathrm{x}}$

Figure 12: First principal strain (a) and horizontal slip (b), in the large-scale domain obtained when prescribing the effective slip only at boundary of the RVE. Location of the largest principal strain indicated with dot. 


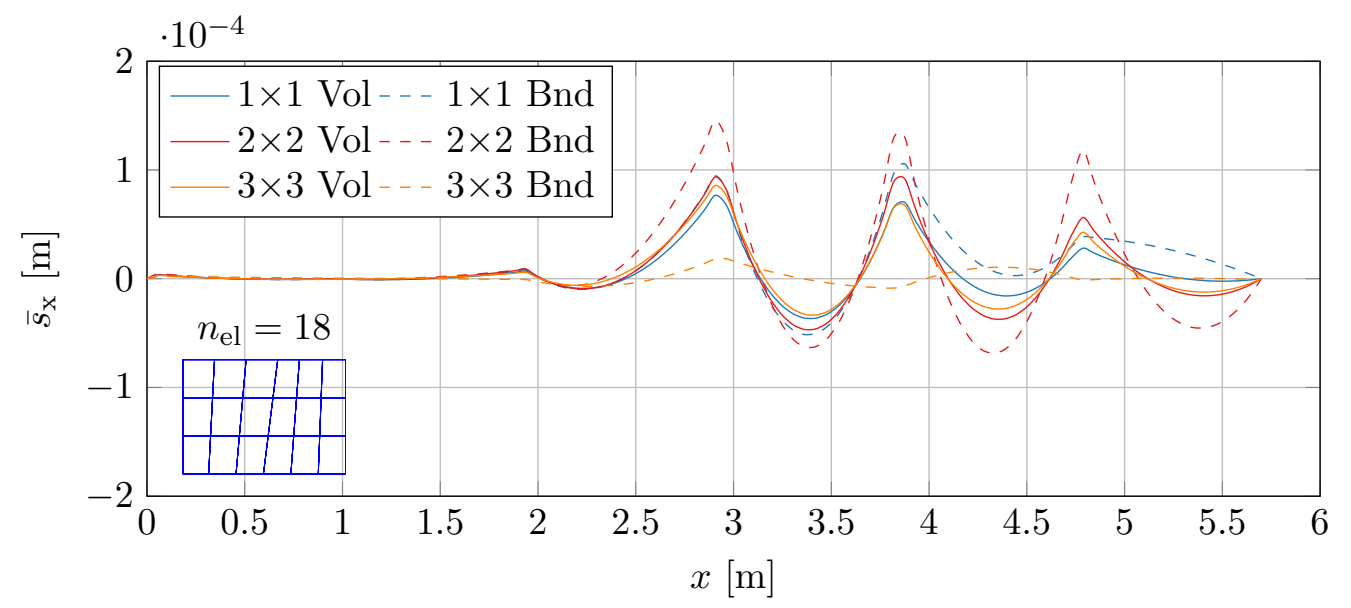

Figure 13: Macroscopic slip profile along the line $(0 \mathrm{~m}, 0.9 \mathrm{~m})--(5.7 \mathrm{~m}, 0.9 \mathrm{~m})$ for volumetric (Vol) and boundary (Bnd) definitions of effective slip. Large-scale mesh with $n_{\mathrm{el}}=18$ elements.

localised effective strain as single macroscopic cracks. If local information, e.g., crack width is sought, the subscale results must be further consulted.

\subsection{Effective slip profiles}

As already mentioned in Section 4, one of the benefits of prescribing the macroscopic slip in the volume was that the physical interpretation of the effective variable was consistent and independent of the unit cell size. Even though it was shown for the local pull-through tests, this effect was not studied globally. In order to further assess this, the slip fields presented in the previous section were studied closer. To this end, macroscopic slip profiles along the line $(0 \mathrm{~m}, 0.9 \mathrm{~m})--(5.7 \mathrm{~m}, 0.9 \mathrm{~m})$ were extracted from the large-scale results in Figures $11 \mathrm{~b}$ and $12 \mathrm{~b}$, and are presented for all large-scale meshes in Figures 13 to 17.

Theoretically, if the physical meaning of the macroscopic variable was independent of the RVE size, the slip fluctuations would ideally have the same amplitude and period. Practically, this can be easily observed only for the coarsest large-scale mesh in Figure 13, where the periods and amplitudes of the macroscopic slip were similar for all unit cells when the slip is prescribed in volume. In case the slip was prescribed only at boundary of the unit cell, the individual amplitudes are almost arbitrary, which signifies that the effective variable must have a different physical interpretation depending on the unit cell size. For finer meshes the picture becomes more complex, but overall it can be said that prescribing the slip in volume produced more consistent effective slip profiles of lower amplitudes than prescribing the slip at boundary. Moreover, it can be observed that on average, prescribing the effective slip in volume of the RVE resulted in smaller amplitudes of the slip (and effective strain), when compared to the case of prescribing the slip at RVE boundary only. It is noteworthy, that the fields were still dependent on the resolution of the large-scale mesh. 


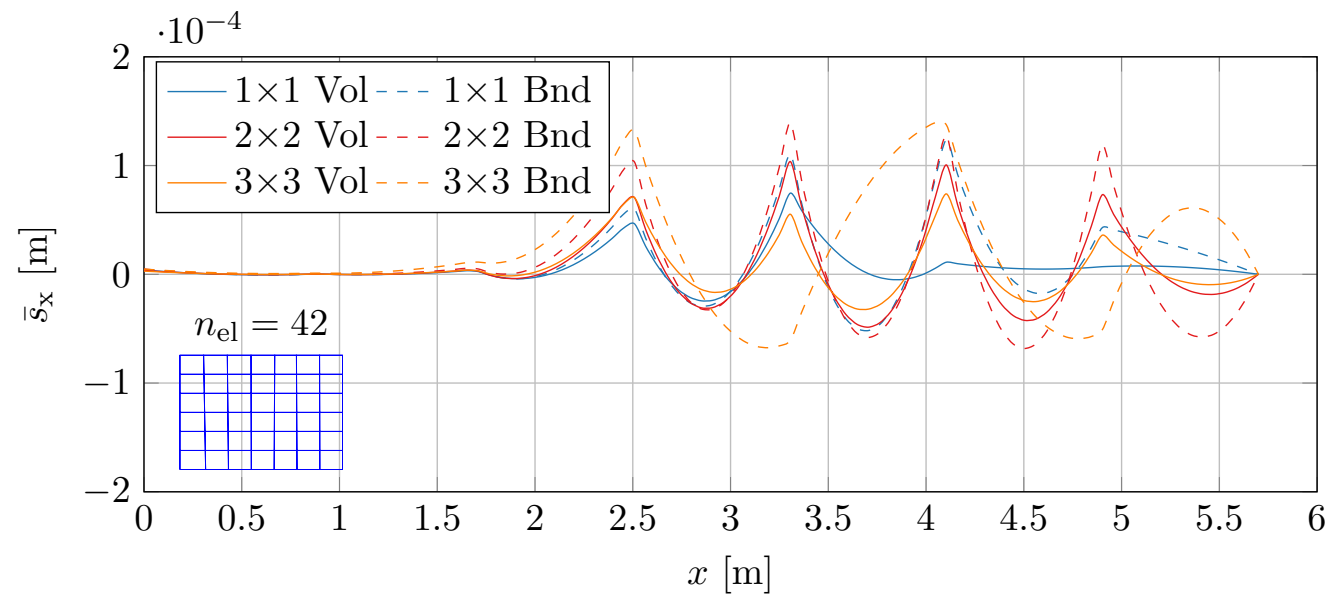

Figure 14: Macroscopic slip profile along the line $(0 \mathrm{~m}, 0.9 \mathrm{~m})--(5.7 \mathrm{~m}, 0.9 \mathrm{~m})$ for volumetric (Vol) and boundary (Bnd) definitions of effective slip. Large-scale mesh with $n_{\mathrm{el}}=42$ elements.

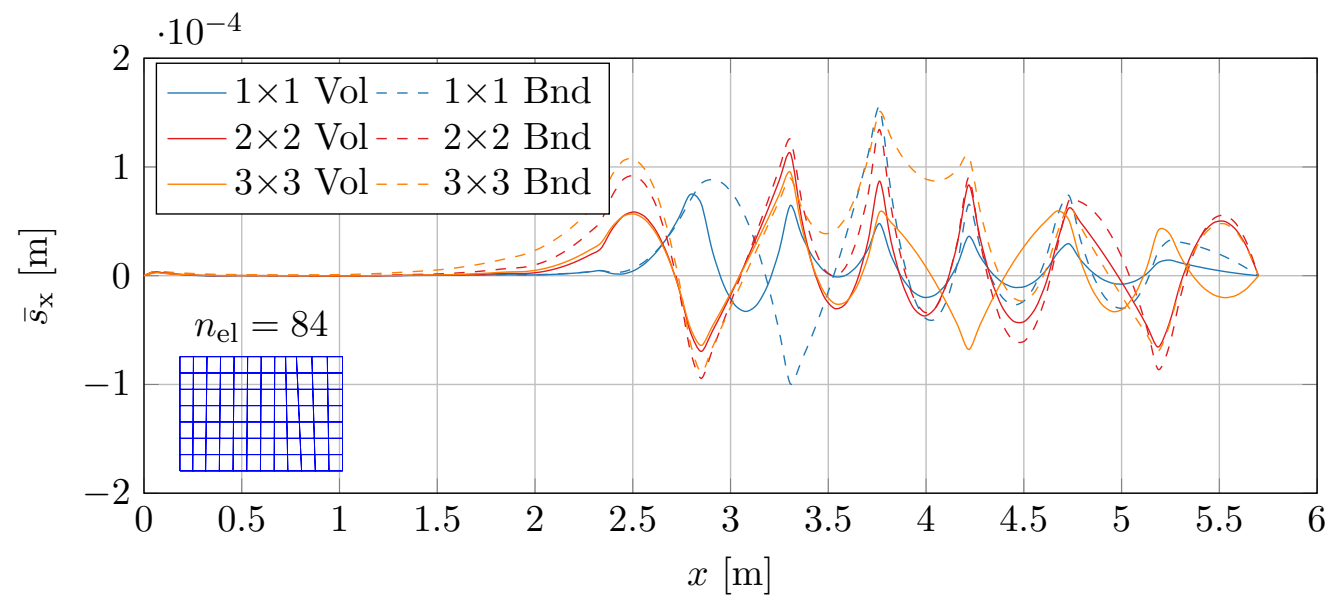

Figure 15: Macroscopic slip profile along the line $(0 \mathrm{~m}, 0.9 \mathrm{~m})--(5.7 \mathrm{~m}, 0.9 \mathrm{~m})$ for volumetric (Vol) and boundary (Bnd) definitions of effective slip. Large-scale mesh with $n_{\mathrm{el}}=84$ elements. 


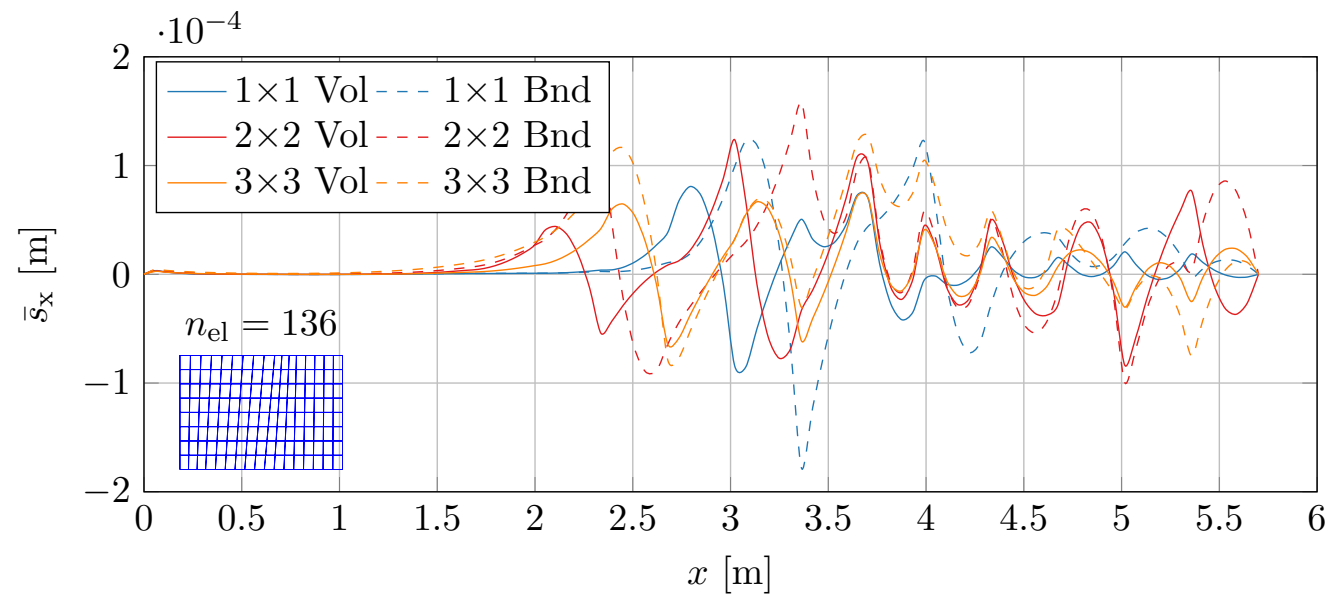

Figure 16: Macroscopic slip profile along the line $(0 \mathrm{~m}, 0.9 \mathrm{~m})--(5.7 \mathrm{~m}, 0.9 \mathrm{~m})$ for volumetric (Vol) and boundary (Bnd) definitions of effective slip. Large-scale mesh with $n_{\mathrm{el}}=136$ elements.

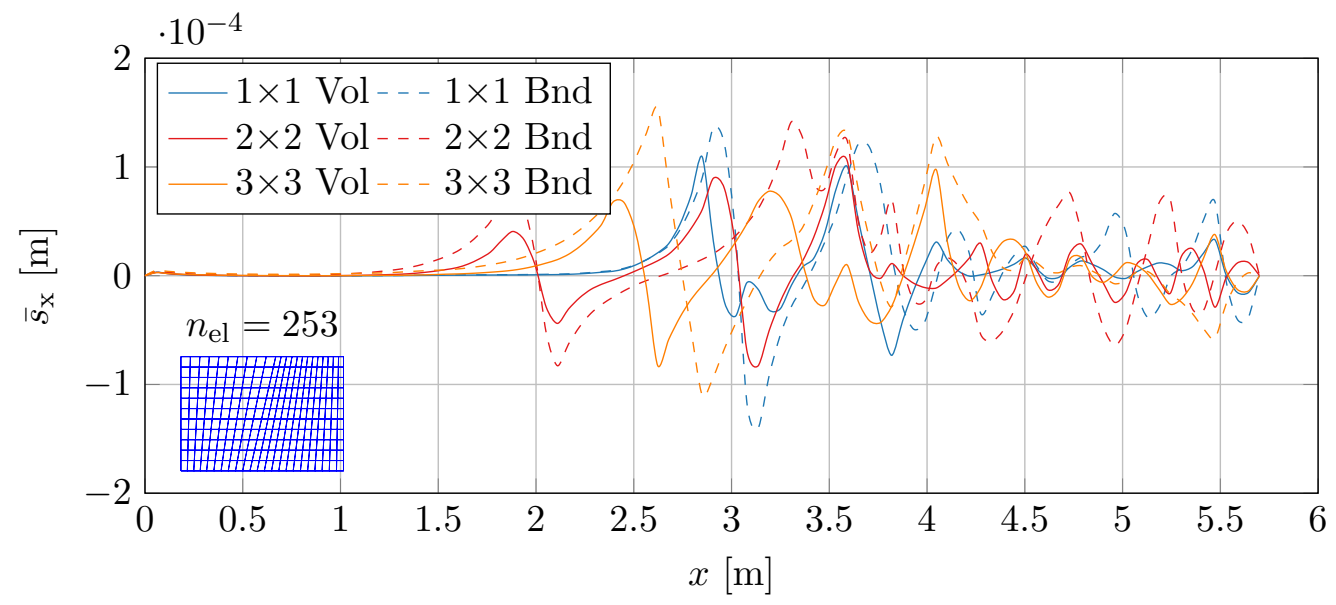

Figure 17: Macroscopic slip profile along the line $(0 \mathrm{~m}, 0.9 \mathrm{~m})--(5.7 \mathrm{~m}, 0.9 \mathrm{~m})$ for volumetric (Vol) and boundary (Bnd) definitions of effective slip. Large-scale mesh with $n_{\mathrm{el}}=253$ elements. 


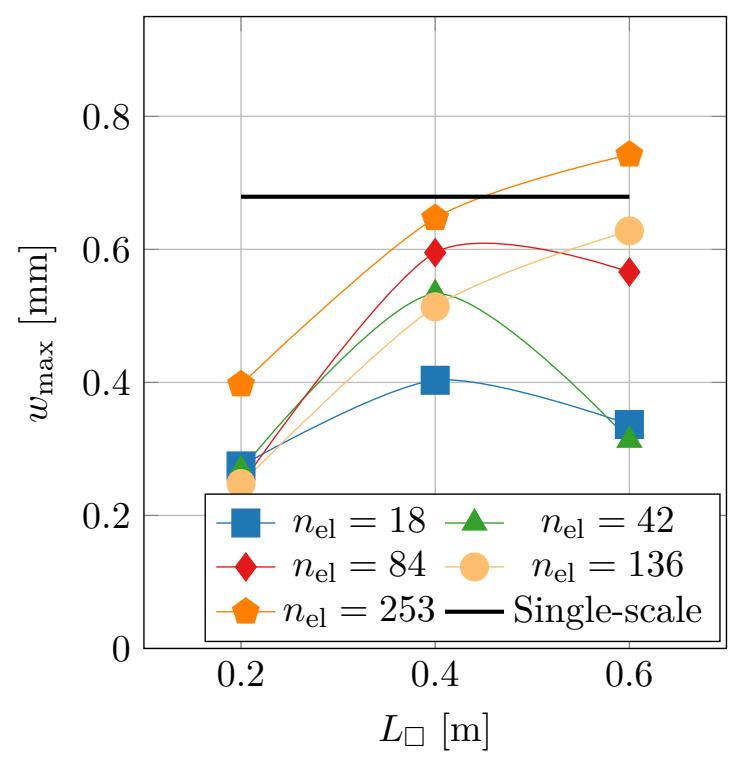

(a) Volumetric definition

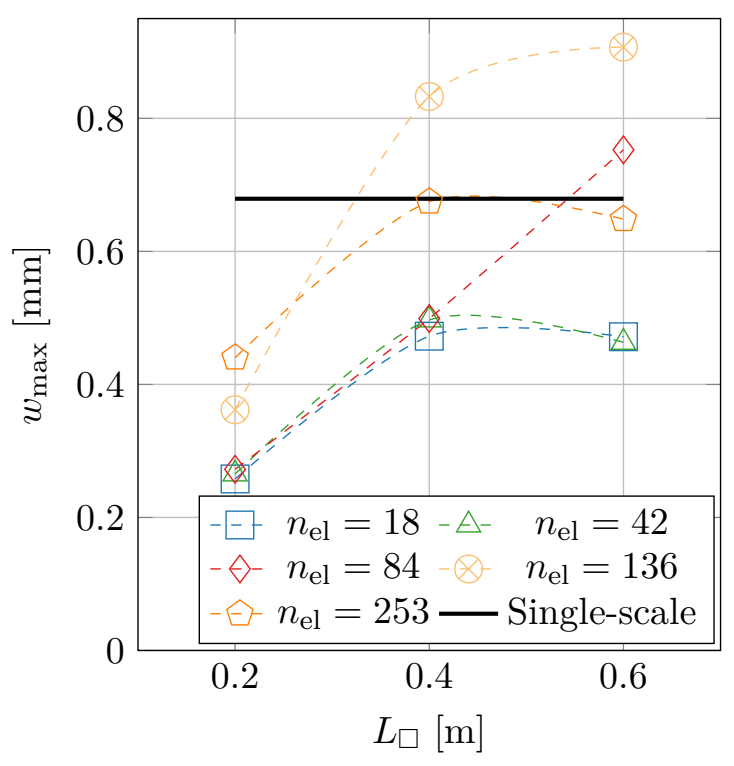

(b) Boundary definition

Figure 18: Maximum crack width for different sizes of unit cell and different macroscopic mesh size obtained with volumetric definition (a) and boundary definition (b) of effective slip.

\subsection{Maximum crack width}

As already mentioned, the ability to predict maximum crack width is of importance in modelling of reinforced concrete structures. This is important especially in serviceability limit state, where the structure can be expected to spend most of its lifetime. To this end, the crack widths produced by the multi-scale model is studied and compared crack widths obtained from a single-scale analysis in full resolution. Even though the crack width in general varies considerably depending on the type of fracture formulation and regularisation with respect to finite element mesh size, the focus of this study is to observe the impact of the computational homogenisation procedure, keeping the constitutive models fixed in both two-scale and single-scale models. Furthermore, the effect of the proposed volumetric formulation is of particular interest. In order to obtain the largest crack width in the twoscale model, the integration point with the largest principal strain is sought. Subscale results in the unit cell are then consulted for the corresponding Gauss point. These results are by default available in all integration points. However, in order to reduce the amount of data, output for only few specific (or none at all) integration points can be requested. In this case, after finding the macroscopic integration point with the largest principal strain, the strain/slip/slip gradient history can be extracted and imposed on a chosen unit cell. Thus, the subscale results can be obtained for an arbitrary macroscopic point in a quick and efficient manner, since a single RVE problem is usually not very demanding in a computational sense.

The maximum crack widths for different large-scale mesh sizes and RVE sizes are presented in Figures 18 and 19 for both volumetric and boundary definition of effective slip. 


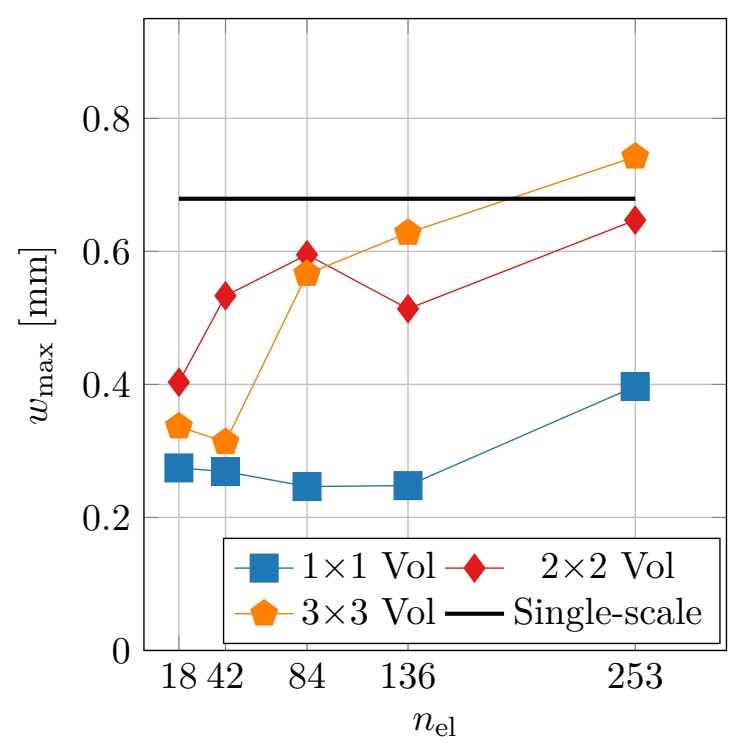

(a) Volumetric definition

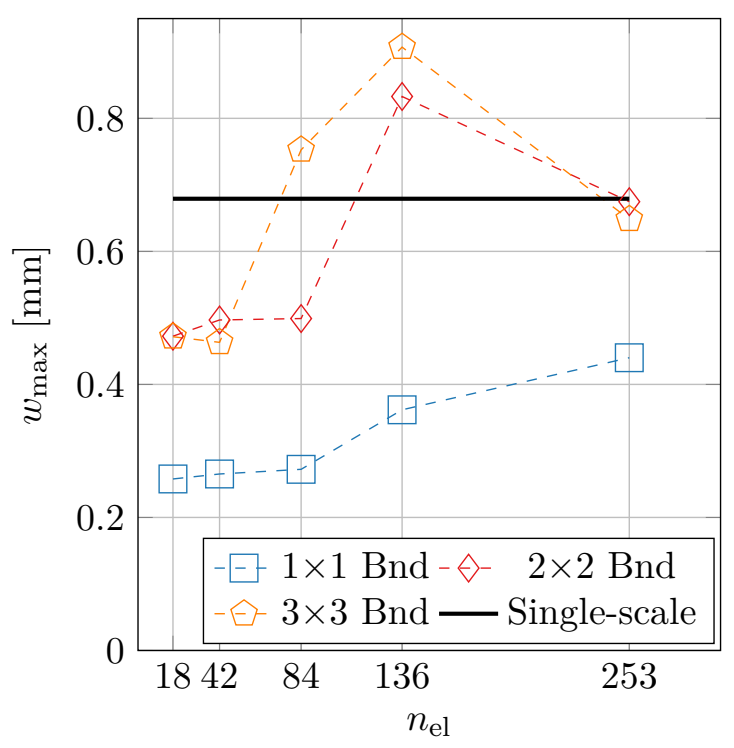

(b) Boundary definition

Figure 19: Maximum crack width for different sizes of unit cell and different macroscopic mesh size obtained with volumetric definition (a) and boundary definition (b) of effective slip.

The crack data pertains to the same load step as in the previous sections. For convenience, the single-scale result was plotted as well. From the results, it is evident that most of the two-scale analyses underestimate the maximum crack width, with only a few exceptions. Overall, it can be observed that it was rather difficult for the smallest unit cell to predict the correct crack width, although the results seem to improve with large-scale mesh refining. Larger RVEs (and possibly finer large-scale meshes) were needed to predict the maximum crack width reasonably well, in this case. However, it can also be observed that the variance of the maximum crack width predictions was lower when the effective slip was prescribed in volume. When the slip was prescribed at the boundary, the spread of the predictions was bigger for the different large-scale meshes, c.f. Figures 18 and 19. This signifies that the volumetric definition results in slightly more consistent predictions of the maximum crack width.

\section{Conclusions}

In this paper, the already existing two-scale model for reinforced concrete with macroscopic variation of reinforced slip was further developed by introducing a novel definition of the effective slip, whereby the slip and its gradient are imposed on the RVE in a weak manner, i.e., by Lagrange multipliers. First, the single-scale structural problem for a reinforced concrete structure was postulated in variational format, and the corresponding two-scale formulation based on Variationally Consistent Homogenisation was derived. It is noteworthy, that not only the concrete, but also the reinforcement slip possessed both a macroscopic 
and fluctuation component. The pertinent large-scale (macroscopic) problem on "effective" single-phase solid was formulated in terms of finding the unknown effective displacement and slip fields. A novel volumetric definition of effective reinforcement slip and its gradient was devised. At the subscale, the concrete, reinforcement and the bond between them was modelled in detail. Standard linear variation of the macroscopic variables was considered for the subscale unit cells, i.e., first-order computational homogenisation was employed. For the displacement in concrete, standard Dirichlet assumption (vanishing fluctuations at boundary) was chosen, while the effective slip and its gradient were prescribed on the Representative Volume Element (RVE) weakly via Lagrange multipliers, which were shown to correspond to the effective work conjugates associated with the macroscopic slip and its gradient.

The effect of the newly devised macroscopic slip definition was studied by means of reinforcement pull-through tests in the subscale unit cells. It was shown that the new volumetric definition solved the problem of rapidly decreasing slip in the interior of reinforcement bars, which was prevalent when the macroscopic slip was imposed only at the boundary of the RVE. In the new formulation, the slip was uniformly distributed along the bars, even for longer rebars, which resulted in RVE size independent interpretation of both the effective slip and its work conjugate. Furthermore, the effective bond-slip behaviour could be directly recovered even for larger unit cells, which was not the case for the former boundary type formulation.

To study the influence on the global behaviour, a uniformly reinforced deep beam was analysed with the multiscale method. The type of macroscopic definition did not have much influence on the force-mid-span deflection relation. Effective strain localisation was obtained for both volumetric and boundary definitions of macroscopic slip, with the volumetric definition producing slightly lower amplitudes of effective strain and slip. Macroscopic slip profiles showed that prescribing the slip in RVE volume resulted in more consistent amplitude of effective slip fluctuations. Maximum crack widths were underestimated by most multiscale analyses. At the same time, the predictions were more consistent for the volumetric definition of slip, i.e., the variance between individual crack predictions was smaller than when the slip was prescribed only at RVE boundary.

The main benefit of the proposed volumetric formulation for the effective slip is the fact that an RVE size independent physical interpretation of the macroscopic slip and its work conjugate, is obtained. This can be useful if effective bond-slip properties of uniformly reinforced concrete are of interest.

For the future work, the two-scale model should be further developed for use in three dimensions, in order to be able to model a greater variety of reinforced concrete structures. In this setting, it is important to study three-dimensional reinforced concrete unit cells. At the large-scale, a first step towards full three-dimensional setting would be to consider computational homogenisation to effective plate/shell elements.

\section{Acknowledgements}

This research was financially supported by the Swedish Research Council (Vetenskapsrådet) under Grant 621-2014-5168, which is gratefully acknowledged. The computations 
were performed on resources at the Chalmers Centre for Computational Science and Engineering (C3SE) provided by the Swedish National Infrastructure for Computing (SNIC).

\section{References}

[1] F. Nilenius, F. Larsson, K. Lundgren, K. Runesson, Macroscopic diffusivity in concrete determined by computational homogenization, International Journal for Numerical and Analytical Methods in Geomechanics 37 (11) (2013) 1535-1551. doi:10.1002/nag. 2097.

[2] F. Nilenius, F. Larsson, K. Lundgren, K. Runesson, Computational homogenization of diffusion in three-phase mesoscale concrete, Computational Mechanics 54 (2) (2014) 461-472. doi:10.1007/ s00466-014-0998-0.

[3] F. Nilenius, F. Larsson, K. Lundgren, K. Runesson, Mesoscale modelling of crack-induced diffusivity in concrete, Computational Mechanics 55 (2) (2015) 359-370. doi:10.1007/s00466-014-1105-2.

[4] Fib, Bond of reinforcement in concrete: state-of-the-art report, Bulletin 10 (fib Fédération internationale du béton), International Federation for Structural Concrete, 2000.

[5] A. Daoud, O. Maurel, C. Laborderie, 2D mesoscopic modelling of bar-concrete bond, Engineering Structures 49 (2013) 696-706. doi:10.1016/j.engstruct.2012.11.018.

[6] Fib, Model Code for Concrete Structures 2010, Wiley-VCH Verlag GmbH \& Co. KGaA, 2013.

[7] N. Domínguez, M. A. Fernández, A. Ibrahimbegovic, Enhanced solid element for modelling of reinforced concrete structures with bond-slip, Computers and Concrete 7 (4) (2010) 347-364.

[8] A. Ibrahimbegovic, A. Boulkertous, L. Davenne, D. Brancherie, Modelling of reinforced-concrete structures providing crack-spacing based on X-FEM, ED-FEM and novel operator split solution procedure, International Journal for Numerical Methods in Engineering 83 (4) (2010) 452-481. doi: 10.1002/nme. 2838.

[9] D. Floros, O. A. Ingason, Modelling and simulation of reinforced concrete beams - Coupled analysis of imperfectly bonded reinforcement in fracturing concrete, Master's thesis, Chalmers University of Technology (2013).

[10] J. F. Unger, S. Eckardt, Multiscale Modeling of Concrete, Archives of Computational Methods in Engineering 18 (3) (2011) 341-393. doi:10.1007/s11831-011-9063-8.

[11] T. Belytschko, J.-H. Song, Coarse-graining of multiscale crack propagation, International Journal for Numerical Methods in Engineering 81 (5) (2010) 537-563. doi:10.1002/nme.2694.

[12] K. Matouš, M. G. Geers, V. G. Kouznetsova, A. Gillman, A review of predictive nonlinear theories for multiscale modeling of heterogeneous materials, Journal of Computational Physics 330 (2017) 192-220. doi:10.1016/j.jcp.2016.10.070.

[13] T. Wu, P. Wriggers, Multiscale diffusion-thermal-mechanical cohesive zone model for concrete, Computational Mechanics 55 (5) (2015) 999-1016. doi:10.1007/s00466-015-1149-y.

[14] P. Wriggers, S. O. Moftah, Mesoscale models for concrete: Homogenisation and damage behaviour, Finite Elements in Analysis and Design 42 (7) (2006) 623-636. doi:10.1016/j.finel.2005.11.008.

[15] V. P. Nguyen, M. Stroeven, L. J. Sluys, Multiscale failure modeling of concrete: Micromechanical modeling, discontinuous homogenization and parallel computations, Computer Methods in Applied Mechanics and Engineering 201-204 (2012) 139-156. doi:10.1016/j.cma.2011.09.014.

[16] A. Karamnejad, V. P. Nguyen, L. J. Sluys, A multi-scale rate dependent crack model for quasibrittle heterogeneous materials, Engineering Fracture Mechanics 104 (2013) 96-113. doi:10.1016/ j.engfracmech.2013.03.009.

[17] E. A. Rodrigues, O. L. Manzoli, L. A. G. Bitencourt, T. N. Bittencourt, M. Sánchez, An adaptive concurrent multiscale model for concrete based on coupling finite elements, Computer Methods in Applied Mechanics and Engineering 328 (2018) 26-46. doi:10.1016/j.cma.2017.08.048.

[18] B. Sun, Z. Li, Multi-scale modeling and trans-level simulation from material meso-damage to structural failure of reinforced concrete frame structures under seismic loading, Journal of Computational Science 12 (2016) 38-50. doi:10.1016/j.jocs.2015.11.003. 
[19] B. Sun, X. Wang, Z. Li, Meso-scale image-based modeling of reinforced concrete and adaptive multiscale analyses on damage evolution in concrete structures, Computational Materials Science 110 (2015) 39-53. doi:10.1016/j.commatsci.2015.07.050.

[20] J.-L. Le, M. DesHarnais, B. Xue, S. D. Pang, H. Du, A two-scale computational model for thermomechanical analysis of reinforced concrete frames, Engineering Structures 105 (2015) 137-151. doi:10.1016/j. engstruct. 2015.09.041.

[21] A. Sciegaj, F. Larsson, K. Lundgren, F. Nilenius, K. Runesson, Two-scale finite element modelling of reinforced concrete structures: Effective response and subscale fracture development, International Journal for Numerical Methods in Engineering 114 (10) (2018) 1074-1102. doi:10.1002/nme.5776.

[22] A. Sciegaj, F. Larsson, K. Lundgren, F. Nilenius, K. Runesson, A multiscale model for reinforced concrete with macroscopic variation of reinforcement slip, Computational Mechanics 63 (2) (2019) 139-158. doi:10.1007/s00466-018-1588-3.

[23] F. Feyel, J.-L. Chaboche, FE2 multiscale approach for modelling the elastoviscoplastic behaviour of long fibre $\mathrm{SiC} / \mathrm{Ti}$ composite materials, Computer Methods in Applied Mechanics and Engineering 183 (3) (2000) 309-330. doi:10.1016/S0045-7825 (99)00224-8.

[24] F. Feyel, A multilevel finite element method (FE2) to describe the response of highly non-linear structures using generalized continua, Computer Methods in Applied Mechanics and Engineering 192 (28) (2003) 3233-3244. doi:10.1016/S0045-7825(03)00348-7.

[25] E. Svenning, F. Larsson, M. Fagerström, Two-scale modeling of fracturing solids using a smeared macro-to-micro discontinuity transition, Computational Mechanics 60 (4) (2017) 627-641. doi:10. $1007 / \mathrm{s} 00466-017-1426-z$.

[26] E. Svenning, F. Larsson, M. Fagerström, A two-scale modeling framework for strain localization in solids: XFEM procedures and computational aspects, Computers and Structures 211 (2019) 43-54. doi: $10.1016 / j$. compstruc.2018.08.003.

[27] J. F. Unger, An FE2-X1 approach for multiscale localization phenomena, Journal of the Mechanics and Physics of Solids 61 (4) (2013) 928-948. doi:10.1016/j.jmps.2012.12.010.

[28] J. Oliver, M. Caicedo, E. Roubin, J. Hernández, A. Huespe, Multi-scale (FE2) analysis of material failure in cement/aggregate-type composite structures, Computational Modelling of Concrete Structures (2014) 39-49.

[29] R. Lackner, H. A. Mang, Scale transition in steel-concrete interaction. I: Model, Journal of Engineering Mechanics 129 (4) (2003) 393-402. doi:10.1061/(ASCE) 0733-9399(2003) 129:4(393).

[30] R. Lackner, H. A. Mang, Scale transition in steel-concrete interaction. II: Applications, Journal of Engineering Mechanics 129 (4) (2003) 403-413. doi:10.1061/(ASCE) 0733-9399(2003) 129:4(403).

[31] F. Larsson, K. Runesson, F. Su, Variationally consistent computational homogenization of transient heat flow, International Journal for Numerical Methods in Engineering 81 (13) (2010) 1659-1686. doi: $10.1002 / \mathrm{nme} .2747$.

[32] M. Geers, V. G. Kouznetsova, W. A. M. Brekelmans, MultiScale first-order and second-order computational homogenization of microstructures towards continua, International Journal for Multiscale Computational Engineering 1 (4) (2003) 371-386. doi:10.1615/Int JMultCompEng.v1.i4.40.

[33] E. Svenning, M. Fagerström, F. Larsson, Computational homogenization of microfractured continua using weakly periodic boundary conditions, Computer Methods in Applied Mechanics and Engineering 299 (2016) 1-21. doi:10.1016/j.cma.2015.10.014.

[34] P. J. Blanco, P. J. Sánchez, E. A. de Souza Neto, R. A. Feijóo, Variational foundations and generalized unified theory of RVE-based multiscale models, Archives of Computational Methods in Engineering 23 (2) (2016) 191-253. doi:10.1007/s11831-014-9137-5.

[35] J. Mazars, A description of micro- and macroscale damage of concrete structures, Engineering Fracture Mechanics 25 (5) (1986) 729-737. doi:10.1016/0013-7944(86)90036-6.

[36] J. Mazars, G. Pijaudier-Cabot, Continuum damage theory - application to concrete, Journal of Engineering Mechanics 115 (2) (1989) 345-365. doi:10.1061/(ASCE) 0733-9399(1989) 115:2(345).

[37] J. Oliver, M. Cervera, S. Oller, J. Lubliner, Isotropic damage models and smeared crack analysis of concrete, in: Computer Aided Analysis and Design of Concrete Structures, 1990, pp. 945-957. 
[38] B. Patzák, OOFEM - an object-oriented simulation tool for advanced modeling of materials and structures, Acta Polytechnica 52 (6) (2012) 59-66.

\section{Appendix A. Scaling tensors}

In order to derive the scaling tensor $\boldsymbol{C}_{\mathrm{s}}$, the first relation in Equation (37) can be further expanded with help of Equations (29)-(30) as

$$
\begin{aligned}
\overline{\boldsymbol{s}}_{\square}\left(\boldsymbol{u}_{\mathrm{c}}^{M}, \boldsymbol{u}_{\mathrm{s}}^{M}\right) & =\overline{\boldsymbol{u}} \cdot\left[\frac{1}{\left|\Omega_{\square}\right|} \int_{\Gamma_{\square, \mathrm{int}}} S_{\mathrm{s}} \boldsymbol{e}_{\mathrm{l}} \otimes \boldsymbol{e}_{\mathrm{l}} \mathrm{d} \Gamma \cdot \boldsymbol{C}_{\mathrm{s}}-\frac{1}{\left|\Omega_{\square}\right|} \int_{\Omega_{\square}} \mathbb{I} \mathrm{d} \Omega\right] \\
& +\overline{\boldsymbol{\varepsilon}}^{T}:\left[\frac{1}{\left|\Omega_{\square}\right|} \int_{\Gamma_{\square, \mathrm{int}}} S_{\mathrm{s}}[\boldsymbol{x}-\overline{\boldsymbol{x}}] \otimes \boldsymbol{e}_{\mathrm{l}} \otimes \boldsymbol{e}_{\mathrm{l}} \mathrm{d} \Gamma \cdot \boldsymbol{C}_{\mathrm{s}}-\frac{1}{\left|\Omega_{\square}\right|} \int_{\Omega_{\square}}[\boldsymbol{x}-\overline{\boldsymbol{x}}] \otimes \mathbb{I} \mathrm{d} \Omega\right] \\
& +\overline{\boldsymbol{s}} \cdot\left[\frac{1}{\left|\Omega_{\square}\right|} \int_{\Gamma_{\square, \mathrm{int}}} S_{\mathrm{s}} \boldsymbol{e}_{\mathrm{l}} \otimes \boldsymbol{e}_{\mathrm{l}} \mathrm{d} \Gamma \cdot \boldsymbol{C}_{\mathrm{s}}\right]+\overline{\boldsymbol{g}}_{\mathrm{d}}^{T}:\left[\frac{1}{\left|\Omega_{\square}\right|} \int_{\Gamma_{\square, \mathrm{int}}} S_{\mathrm{s}}[\boldsymbol{x}-\overline{\boldsymbol{x}}] \otimes \boldsymbol{e}_{\mathrm{l}} \otimes \boldsymbol{e}_{\mathrm{l}} \mathrm{d} \Gamma \cdot \boldsymbol{C}_{\mathrm{s}}\right] .
\end{aligned}
$$

Accounting for $\frac{1}{\left|\Omega_{\square}\right|} \int_{\Omega_{\square}} \boldsymbol{x}-\overline{\boldsymbol{x}} \mathrm{d} \Omega=0$ and setting

$$
\boldsymbol{C}_{\mathrm{s}}=\left|\Omega_{\square}\right|\left[\int_{\Gamma_{\square, \mathrm{int}}} S_{\mathrm{s}} \boldsymbol{e}_{\mathrm{l}} \otimes \boldsymbol{e}_{\mathrm{l}} \mathrm{d} \Gamma\right]^{-1}
$$

the homogenisation-prolongation identity becomes

$$
\overline{\boldsymbol{s}}_{\square}\left(\boldsymbol{u}_{\mathrm{c}}^{M}, \boldsymbol{u}_{\mathrm{s}}^{M}\right)=\overline{\boldsymbol{u}} \cdot \mathbf{0}+\overline{\boldsymbol{s}} \cdot \mathbb{I}+\left[\overline{\boldsymbol{\varepsilon}}+\overline{\boldsymbol{g}}_{\mathrm{d}}\right]^{T}: \underbrace{\left[\frac{1}{\left|\Omega_{\square}\right|} \int_{\Gamma_{\square, \mathrm{int}}} S_{\mathrm{s}}[\boldsymbol{x}-\overline{\boldsymbol{x}}] \otimes \boldsymbol{e}_{\mathrm{l}} \otimes \boldsymbol{e}_{\mathrm{l}} \mathrm{d} \Gamma \cdot \boldsymbol{C}_{\mathrm{s}}\right]}_{\boldsymbol{A}}
$$

Assuming a symmetric layout of reinforcement in the unit cell, $\boldsymbol{A}=\mathbf{0}$. Alternatively, we would have to change $\overline{\boldsymbol{x}} \rightarrow \overline{\boldsymbol{x}}_{\mathrm{s}, 1}, \overline{\boldsymbol{x}}_{\mathrm{s}, 2}$ in Equation (30), so that

$$
u_{\mathrm{s}, 1}^{M}(\overline{\boldsymbol{x}}, \boldsymbol{x})=\boldsymbol{e}_{\mathrm{l}} \cdot[\overline{\boldsymbol{u}}(\overline{\boldsymbol{x}})+\overline{\boldsymbol{s}}(\overline{\boldsymbol{x}})]+\boldsymbol{e}_{\mathrm{l}} \cdot\left[\overline{\boldsymbol{\varepsilon}}(\overline{\boldsymbol{x}})+\overline{\boldsymbol{g}}_{\mathrm{d}}(\overline{\boldsymbol{x}})\right] \cdot\left[\boldsymbol{x}-\overline{\boldsymbol{x}}_{\mathrm{s}, \mathrm{i}}\right] \quad \text { if } \boldsymbol{e}_{\mathrm{l}}=\boldsymbol{e}_{\mathrm{l}, \mathrm{i}}
$$

with

$$
\overline{\boldsymbol{x}}_{\mathrm{s}, \mathrm{i}}=\frac{1}{\left|\Omega_{\square}\right|} \int_{\Gamma_{\square, \mathrm{int}}} S_{\mathrm{s}}[\boldsymbol{x}-\overline{\boldsymbol{x}}] \boldsymbol{e}_{1} \cdot \boldsymbol{e}_{1, \mathrm{i}} \mathrm{d} \Gamma .
$$

In order to derive the scaling tensor $\boldsymbol{C}_{\mathrm{g}}$, we look again at the second relation in Equation (37) and expand it with help of Eqs. (29)-(30) as

$$
\begin{aligned}
\overline{\boldsymbol{g}}_{\mathrm{d}, \square}\left(\boldsymbol{u}_{\mathrm{c}}^{M}, \boldsymbol{u}_{\mathrm{s}}^{M}\right) & =\overline{\boldsymbol{g}}_{\mathrm{d}}^{T}:\left[\frac{1}{\left|\Omega_{\square}\right|} \int_{\Gamma_{\square, \mathrm{int}}} S_{\mathrm{s}} \boldsymbol{e}_{\mathrm{l}} \otimes \boldsymbol{e}_{\mathrm{l}} \otimes \boldsymbol{e}_{\mathrm{l}} \otimes \boldsymbol{e}_{\mathrm{l}} \mathrm{d} \Gamma \cdot \boldsymbol{C}_{\mathrm{g}}\right] \\
& +\overline{\boldsymbol{\varepsilon}}^{T}:\left[\frac{1}{\left|\Omega_{\square}\right|} \int_{\Gamma_{\square, \mathrm{int}}} S_{\mathrm{s}} \boldsymbol{e}_{\mathrm{l}} \otimes \boldsymbol{e}_{\mathrm{l}} \otimes \boldsymbol{e}_{\mathrm{l}} \otimes \boldsymbol{e}_{\mathrm{l}} \mathrm{d} \Gamma \cdot \boldsymbol{C}_{\mathrm{g}}-\sum_{i=1}^{2} \frac{1}{\left|\Omega_{\square}\right|} \int_{\Omega_{\square}} \boldsymbol{e}_{\mathrm{l}, \mathrm{i}} \otimes \boldsymbol{e}_{\mathrm{l}, \mathrm{i}} \otimes \boldsymbol{e}_{\mathrm{l}, \mathrm{i}} \otimes \boldsymbol{e}_{\mathrm{l}, \mathrm{i}} \mathrm{d} \Omega\right]
\end{aligned}
$$


Using that $\boldsymbol{e}_{1} \in\left\{\boldsymbol{e}_{1,1}, \boldsymbol{e}_{1,2}\right\}$ with $\boldsymbol{e}_{1,1} \perp \boldsymbol{e}_{1,2}$, and assuming the shape of scaling as

$$
\boldsymbol{C}_{\mathrm{g}}=\sum_{i=1}^{2} C_{\mathrm{g}, \mathrm{ii}} \boldsymbol{e}_{\mathrm{l}, \mathrm{i}} \otimes \boldsymbol{e}_{\mathrm{l}, \mathrm{i}}
$$

we may express the integrals as

$$
\begin{aligned}
\overline{\boldsymbol{g}}_{\mathrm{d}, \square}\left(\boldsymbol{u}_{\mathrm{c}}^{M}, \boldsymbol{u}_{\mathrm{s}}^{M}\right) & =\sum_{i=1}^{2} \bar{g}_{\mathrm{d}, \mathrm{ii}} \boldsymbol{e}_{\mathrm{l}, \mathrm{i}} \otimes \boldsymbol{e}_{\mathrm{l}, \mathrm{i}}\left[\frac{1}{\left|\Omega_{\square}\right|} \int_{\Gamma_{\square, \mathrm{int}}} S_{\mathrm{s}} \boldsymbol{e}_{\mathrm{l}} \cdot \boldsymbol{e}_{\mathrm{l}, \mathrm{i}} \mathrm{d} \Gamma C_{\mathrm{g}, \mathrm{ii}}\right] \\
+ & \sum_{i=1}^{2} \bar{\varepsilon}_{\mathrm{ii}} \boldsymbol{e}_{\mathrm{l}, \mathrm{i}} \otimes \boldsymbol{e}_{\mathrm{l}, \mathrm{i}}[\frac{1}{\left|\Omega_{\square}\right|} \int_{\Gamma_{\square, \mathrm{int}}} S_{\mathrm{s}} \boldsymbol{e}_{\mathrm{l}} \cdot \boldsymbol{e}_{\mathrm{l}, \mathrm{i}} \mathrm{d} \Gamma C_{\mathrm{g}, \mathrm{ii}}-\underbrace{\frac{1}{\left|\Omega_{\square}\right|} \int_{\Omega_{\square}} \mathrm{d} \Omega}_{=1}] .
\end{aligned}
$$

We are now in position to assure that

$$
\overline{\boldsymbol{g}}_{\mathrm{d}, \square}\left(\boldsymbol{u}_{\mathrm{c}}^{M}, \boldsymbol{u}_{\mathrm{s}}^{M}\right)=\sum_{i=1}^{2} \bar{g}_{\mathrm{d}, \mathrm{ii}} \boldsymbol{e}_{\mathrm{l}, \mathrm{i}} \otimes \boldsymbol{e}_{\mathrm{l}, \mathrm{i}}=\overline{\boldsymbol{g}}_{\mathrm{d}},
$$

if

$$
C_{\mathrm{g}, \mathrm{ii}}=\left|\Omega_{\square}\right|\left[\int_{\Gamma_{\square, \mathrm{int}}} S_{\mathrm{s}} \boldsymbol{e}_{\mathrm{l}} \cdot \boldsymbol{e}_{\mathrm{l}, \mathrm{i}} \mathrm{d} \Gamma\right]^{-1} .
$$

Moreover, is is noteworthy that

$$
\boldsymbol{C}_{\mathrm{g}}=\boldsymbol{C}_{\mathrm{s}}=\sum_{i=1}^{2} \boldsymbol{e}_{\mathrm{l}, \mathrm{i}} \otimes \boldsymbol{e}_{\mathrm{l}, \mathrm{i}}\left|\Omega_{\square}\right|\left[\int_{\Gamma_{\square, \mathrm{int}}} S_{\mathrm{s}} \boldsymbol{e}_{\mathrm{l}} \cdot \boldsymbol{e}_{\mathrm{l}, \mathrm{i}} \mathrm{d} \Gamma\right]^{-1}
$$

is diagonal. 